\title{
OPEN A genetic sum score of effect alleles associated with serum lipid concentrations interacts with educational attainment
}

\author{
Carina Emmel ${ }^{1 \bowtie}$, Mirjam Frank ${ }^{1}$, Nico Dragano ${ }^{2}$, Markus M. Nöthen ${ }^{3,4}$, Raimund Erbel ${ }^{1}$, \\ Karl-Heinz Jöckel ${ }^{1}$ \& Börge Schmidt ${ }^{1}$
}

High-density lipoprotein cholesterol (HDL-C), low-density lipoprotein cholesterol (LDL-C), and total cholesterol (TC) levels are influenced by both genes and the environment. The aim was to investigate whether education and income as indicators of socioeconomic position (SEP) interact with lipidincreasing genetic effect allele scores (GES) in a population-based cohort. Using baseline data of 4516 study participants, age- and sex-adjusted linear regression models were fitted to investigate associations between GES and lipids stratified by SEP as well as including GES $\times$ SEP interaction terms. In the highest education group compared to the lowest stronger effects per GES standard deviation were observed for HDL-C (2.96 mg/dl [95\%-Cl: $2.19,3.83]$ vs. $2.45 \mathrm{mg} / \mathrm{dl}$ [95\%-Cl: $1.12,3.72])$, LDL-C $(6.57 \mathrm{mg} / \mathrm{dl}[95 \%-\mathrm{Cl}: 4.73,8.37]$ vs. $2.66 \mathrm{mg} / \mathrm{dl}[95 \%-\mathrm{Cl}:-0.50,5.76])$ and TC $(8.06 \mathrm{mg} / \mathrm{dl}[95 \%-\mathrm{Cl}:$ $6.14,9.98]$ vs. $4.37 \mathrm{mg} / \mathrm{dl}$ [95\%-Cl: $0.94,7.80]$ ). Using the highest education group as reference, interaction terms showed indication of GES by low education interaction for LDL-C ( $\beta_{\text {GES XEducation: }}$ : $-3.87 ; 95 \%-\mathrm{Cl}:-7.47,-0.32)$, which was slightly attenuated after controlling for $\mathrm{GES}_{\mathrm{LDL}-\mathrm{C}} \times$ Diabetes

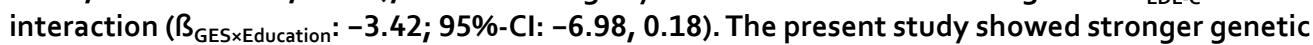
effects on LDL-C in higher SEP groups and gave indication for a GES LDL-C $\times E_{\text {ducation interaction, }}$ demonstrating the relevance of SEP for the expression of genetic health risks.

Elevated serum concentration of low-density lipoprotein cholesterol (LDL-C) is an important causal risk factor for cardiovascular disease (CVD) ${ }^{1-3}$ and has now largely replaced total cholesterol (TC) as the primary treatment target for dyslipidemia ${ }^{1}$. Reduced serum concentration of high-density lipoprotein cholesterol (HDL-C) is independently associated with $\mathrm{CVD}^{4,5}$, in genetic studies however, HDL-C has not been causally associated with $\mathrm{CVD}^{3}$. Serum lipid concentrations (in the following named as lipids) are complex human traits that are influenced by both genetic and lifestyle factors ${ }^{6}$. With regard to health inequalities, research has shown that indicators of socioeconomic position (SEP) such as educational attainment, household income and employment status are also associated with lipids ${ }^{7-11}$. However, the association has been of different strength with regard to different SEP indicators, heterogeneous between men and women and appears to be of different direction in developed and developing countries. In developed countries a more unfavorable serum lipid profile (higher LDL-C and TC levels and lower HDL-C levels) is observed in lower SEP groups ${ }^{8,10-13}$.

The genome-wide association study (GWAS) meta-analysis from the Global Lipids Genetics Consortium (GLGC) in 2013 identified 157 loci associated with lipids accounting for up to $12 \%$ of the variance in each lipid trait $^{14}$. Subsequent large-scale GWAS demonstrated enhanced gene discovery from expanded sample sizes, independently reporting additional novel loci in different ancestry groups, accounting for up to $20 \%$ of the variance in lipids ${ }^{15-17}$. In contrast family-based association studies have indicated that $30-70 \%$ of the variance in lipids is genetically based ${ }^{18,19}$.

It is assumed that gene-environment $(\mathrm{G} \times \mathrm{E})$ interactions, where the effect of some gene variants depend on specific environmental exposure ${ }^{20}$, may account for parts of the unexplained variance of lipids ${ }^{21}$. G $\times \mathrm{E}$ interactions for lipids have been observed for life style risk factors such as obesity, smoking, diet, physical activity,

${ }^{1}$ Institute for Medical Informatics, Biometry and Epidemiology, University Hospital of Essen, University of Duisburg-Essen, Hufelandstraße 55, 45147 Essen, Germany. ${ }^{2}$ Institute of Medical Sociology, Centre for Health and Society, University Hospital Düsseldorf, Düsseldorf, Germany. ${ }^{3}$ Institute of Human Genetics, University of Bonn, Bonn, Germany. ${ }^{4}$ Department of Genomics, Life and Brain Center, University of Bonn, Bonn, Germany. ${ }^{\square}$ email: Carina.emmel@uk-essen.de 
diabetes and sleep duration ${ }^{22-29}$. While some studies showed stronger effects of lipid-increasing alleles in groups reporting a more favorable life style such as lean or physically active individuals ${ }^{27,28}$, Bentley et al. also reported SNPs with stronger effects in smokers as an example for an unfavorable life style ${ }^{24}$. So far interactions of lipidrelated effect alleles with SEP have not been investigated in adults, but GXSEP interactions on overall health and other traits such as body mass index (BMI) have been indicated by twin and population based studies ${ }^{30-32}$. $\mathrm{Li}$ et al. reported an interaction between one SNP related with a metabolically obese, normal weight phenotype including high TC levels and a composite score including parental education and household income on metabolically obese, normal weight children ${ }^{33}$.

SEP can be considered as a context-defining variable that describes certain risk constellations such as unequally distributed environmental, psychosocial and behavioral health risk factors and may be better suited for describing health-related environments as a whole rather than single risk factors. Thus, investigating interactions between lipid-associated loci and SEP indicators may be crucial for identifying subgroups for which genetic effects show stronger signals than for the average population ${ }^{34}$ and who may benefit from genotype-based targeted intervention ${ }^{35}$.

The aim of this study was to investigate whether the SEP indicators education and income interact with genetic sum scores of lipid-increasing effect alleles (GES) for HDL-C $\left(\mathrm{GES}_{\mathrm{HDL}-\mathrm{C}}\right), \mathrm{LDL}_{\mathrm{C}} \mathrm{C}\left(\mathrm{GES}_{\mathrm{LDL}-\mathrm{C}}\right)$ and TC $\left(\mathrm{GES}_{\mathrm{TC}}\right)$ in a population-based cohort study. In order to explore whether any detected SEP interactions can be explained by SEP-associated life style risk factors, information on smoking, BMI, physical activity, alcohol consumption and diabetes mellitus was included in the interaction analysis. Main results of GES based on the GLGC GWAS meta-analysis ${ }^{14}$ were compared to the results of GES also including loci of more recently published GWAS to check for differences in lipid prediction and GES $\times$ SEP interaction.

\section{Methods}

Study population. All analysis are based on baseline data of the Heinz Nixdorf Recall Study (Risk factors evaluation of coronary calcium and lifestyle cohort), a population-based prospective cohort study. Details on the rationale of the study, study design, sampling methods, response rate, and data collection have been published in detail previously ${ }^{36,37}$. In brief, 4814 participants aged 45-74 years were randomly selected between 2000 and 2003 from mandatory registries of residence of the cities Bochum, Essen and Mülheim/Ruhr within the densely populated Ruhr metropolitan area in Germany. All participants gave written informed consent. The study has been approved by the institutional ethics committee of the University Duisburg-Essen and was conducted according to the guidelines and recommendations for ensuring Good Epidemiological Practice ${ }^{38}$. An extended quality management procedure and certification according to DIN ISO 9001:2000 was established. All study participants were of European ancestry and thus genetically very homogeneous.

Data collection. At study baseline, standard enzymatic methods (homogeneous direct determination with OPERA measuring system) were used to measure HDL-C, LDL-C and TC within $12 \mathrm{~h}$ after blood serum collection at the central laboratory of the University Hospital of Essen, Germany. Participants were asked to fast for at least $4 \mathrm{~h}$ before examination resulting in $60 \%$ of subjects with fasting status $>8 \mathrm{~h}, 2 \% 6-8 \mathrm{~h}, 5 \% 4-6 \mathrm{~h}, 26 \%$ $2-4 \mathrm{~h}$ and $7 \%$ with $<2 \mathrm{~h}$ of fasting. The fasting duration was on average $9.7 \mathrm{~h}(\mathrm{SD}: \pm 4.9 \mathrm{~h})$, with no difference between men and women ${ }^{39}$. Information on educational attainment, household income, smoking status, physical activity and diagnosis of diabetes mellitus or use of anti-diabetic medication was collected at study baseline in standardized computer-assisted face-to-face interviews. Information on alcohol consumption was collected with a self-administered questionnaire. Education was defined by combining school and vocational training as total years of formal education according to the International Standard Classification of Education (ISCED $97)^{40}$. Years of education were categorized into three groups with $\leq 10$ years (equivalent to a minimum compulsory school attendance with no additional vocational degree), 11-13 years (equivalent to upper secondary educational degrees or a combination of lower secondary education and vocational training), and $\geq 14$ years of education (equivalent to a vocational training including additional qualification or a university degree). Income was measured as the monthly household equivalent income calculated by dividing the participants' household net income by a weighting factor for each household member ${ }^{41}$. Income was categorized into three groups using sex-specific tertiles. In order to take account for their different mechanisms in causing health inequalities, both SEP indicators were analyzed separately ${ }^{42,43}$. The body-mass-index (BMI) was calculated based on standardized measurements of body weight (in underwear) and height $\left(\mathrm{kg} / \mathrm{m}^{2}\right)$. Physical activity was defined as exercising one and more times per week versus no weekly engagement in physical exercise. Smoking status was dichotomized for analyses as current smoker (smoking cigarettes during the past 12 months) versus former and never smoker. Alcohol intake was estimated as gram of pure alcohol per week using information on the number of alcoholic drinks usually consumed in a week by type of drink (i.e., beer, wine, sparkling wine, spirits). Participants were classified as diabetics if they reported a diagnosis of diabetes mellitus, or if a fasting blood glucose level $\geq 126 \mathrm{mg} / \mathrm{dl}$, a postprandial blood glucose level $\geq 200 \mathrm{mg} / \mathrm{dl}$ was found, or if the use of anti-diabetic medication was documented. All variables have been checked in an ongoing data quality control during the baseline examination period.

Genetic data. Lymphocyte DNA was isolated from EDTA anti-coagulated venous blood using the Chemagic Magnetic Separation Module I (Chemagen, Baesweiler, Germany). Genotyping was performed by matrixassisted laser desorption ionization-time of flight mass spectrometry-based iPLEX Gold assay at the Department of Genomics, Life and Brain Center, Bonn Germany using two different Illumina microarrays (Metabochip, Global Screening Array (GSA); Illumina, San Diego, USA) according to the manufacturer's protocols. Genotype imputation was carried out using IMPUTE v.2.3.0 ${ }^{44}$. Quality control was applied prior to imputation and per- 
formed on subject level including sex-, ethnicity- and relatedness-checks, excluding subjects with missing genotype data $>10 \%$. Further, single nucleotide polymorphisms (SNPs) with a missing genotype frequency $>10 \%$ were excluded.

Using the GLGC GWAS meta-analyses ${ }^{14}$ and all afterwards published large-scale GWAS ${ }^{15-17}, 152$ HDL-C-, 108 LDL-C- and 131 TC-associated SNPs at genome-wide significance level of $p<5 \times 10^{-8}$ in study populations of European ancestry have been selected for analysis (Supplementary Table S1). Of these, 102, 85, and 103 genotyped SNPs or a proxy were found for HDL-C, LDL-C and TC, respectively, on the Metabochip. Additionally, 3/2/3 HDL-C/LDL-C/TC SNPs were found in imputed data of the Metabochip and 18/8/11 HDL-C/LDL-C/TC SNPs or a proxy were found on the GSA (Supplementary Table S1-S4). A proxy was defined as a SNP within a linkage disequilibrium (LD) $\geq 0.8$. For all SNPs included in the analysis, no deviation from Hardy-Weinberg equilibrium was found $\left(p \leq 1 \times 10^{-6}\right)$. LD-based SNP pruning was performed with PLINK to exclude selected SNPs correlated with an $\mathrm{LD} \geq 0.8$ before calculating the $\mathrm{GES}_{\text {Lipid. }}$. However, all SNPs included represented independent loci and none of the SNPs had to be pruned out. Two different GES $_{\text {Lipid }}$ were calculated for each lipid trait: $\mathrm{GES}_{\text {Lipid }}$ based on ${ }^{14}$ and an extended GES $\mathrm{Lipid-EXT}_{\text {based on }}{ }^{14-17}$. In detail, the genetic sum scores of effect alleles for HDL-C $\left(\mathrm{GES}_{\mathrm{HDL}-\mathrm{C}}\right)$, LDL-C $\left(\mathrm{GES}_{\mathrm{LDL}-\mathrm{C}}\right)$ and TC $\left(\mathrm{GES}_{\mathrm{TC}}\right)$ were calculated by aggregating the total number of lipid-increasing effect alleles ( 0,1 or 2$)$ for each individual from the Heinz Nixdorf Recall Study population across the selected SNPs based on the GLGC GWAS meta-analyses ${ }^{14}$. For comparison, extended GES Lipid-EXT $_{\text {have been }}$ calculated for each trait by additionally adding the number of lipid-increasing effect alleles of selected SNPs based on all afterwards published large-scale GWAS (i.e., $\mathrm{GES}_{\text {HDL-C-EXT }}, \mathrm{GES}_{\text {LDL-C-EXT }}, \mathrm{GES}_{\text {TC-EXT) }}{ }^{15-17}$. Imputation of missing genotype information was based on the study sample's effect allele frequencies according to the PLINK scoring routine ${ }^{45}$.

Statistical analyses. Out of the study population $(\mathrm{n}=4814)$ all participants without genetic information $(n=296)$ and missing values for all three lipids (HDL-C, LDL-C and TC) $(n=22)$ were excluded from the analysis, leading to an analysis population of 4516 study participants (50.0\% women) (Supplementary Figure S1). Participants with missing information on education $(n=13)$ and income $(n=283)$, were excluded from respective analysis. Compared to the analysis population, participants with missing genetic information as well as missing values in lipids and SEP indicators did not differ substantially regarding the main variables included in the analysis.

First, sex- and age-adjusted linear regression models were fitted to calculate effect size estimates and their corresponding 95\% confidence intervals (95\% CIs) for the association of education, income and the respective

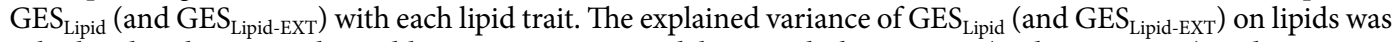
calculated with a non-adjusted linear regression model. Second, the $\mathrm{GES}_{\text {Lipid }}$ (and GES $\mathrm{Lipid-EXT}_{\text {L }}$ ) and SEP main effects as well as GES $\times$ SEP interaction terms were included into sex- and age-adjusted linear regression models to investigate GES $\times$ SEP interactions. Third, the genetic effect of $\mathrm{GES}_{\text {Lipid }}$ (and $\mathrm{GES}_{\text {Lipid-EXT }}$ ) on each lipid trait was calculated stratified by education groups, income tertiles and diabetes status. Fourth, all possible combinations of $\mathrm{GES}_{\text {Lipid }}$ (and $\mathrm{GES}_{\text {Lipid-EXT }}$ ) tertiles and SEP groups were entered into regression models as dummy variables to calculate single reference joint effects of the GES and the SEP indicators, using the group with the highest SEP and the lowest $\mathrm{GES}_{\mathrm{LDL}-\mathrm{C} / \mathrm{TC}}$ tertile and accordingly the highest SEP and the highest GES $\mathrm{HDL}_{\mathrm{C}}$ tertile as reference. Additionally, absolute measures of lipids in each of the different combinations of SEP groups and GES $_{\text {Lipid }}$ tertiles were calculated. Fifth, to analyze whether GES $\times$ SEP interactions may be affected by underlying interactions between GES $_{\text {Lipid }}$ and SEP-related life style risk factors, smoking (S), BMI, physical activity (PA), alcohol consumption (A) or diabetes mellitus (D) main effects and the respective $\mathrm{GES} \times \mathrm{S} / \mathrm{BMI} / \mathrm{PA} / \mathrm{A} / \mathrm{D}$ interaction terms in addition to an $\mathrm{SEP} \times \mathrm{S} / \mathrm{BMI} / \mathrm{PA} / \mathrm{A} / \mathrm{D}$ interaction term were included in the interaction model separately for each life style risk factor. Single SNP main effect and single SNP interaction analysis between SNPs and education groups were performed for all SNPs used in the $\mathrm{GES}_{\text {Lipid. }}$. Education is entered as a dummy variable and only the results of the lowest education group compared to the highest education group as reference were presented. Additionally, participants with lipid-lowering medication $(n=557)$ were considered in sensitivity analyses by (1) adjusting the main results of $\mathrm{GES}_{\text {Lipid }}$, education and GES $\times$ Education for lipid-lowering medication and by (2) excluding individuals with lipid-lowering medication. The LD-based pruning and the calculation of GES and single SNP analyses were performed with using PLINK v1.07 software package ${ }^{45}$ and RStudio v3.6.0 ${ }^{46}$. For all other analyses SAS software v9.4 ${ }^{47}$ was used. All GES Lipid $^{-}$and extended $\mathrm{GES}_{\text {Lipid-EXT-related beta coefficients and } 95 \% \text { CIs (except }}$ for the single reference joint effect analysis) were standardized by multiplying the coefficients by the standard deviation of the respective $\mathrm{GES}_{\text {Lipid }}$ or the respective extended $\mathrm{GES}_{\text {Lipid-EXT }}$ to facilitate comparability of each GES.

\section{Results}

In the analysis population, the mean age ( \pm standard deviation) was $59.6 \pm 7.8$ years and the mean serum lipid concentration ( \pm standard deviation) were $58.16 \pm 17.29 \mathrm{mg} / \mathrm{dl}$ for HDL-C, $145.41 \pm 36.21 \mathrm{mg} / \mathrm{dl}$ for LDL-C and $229.26 \pm 39.18 \mathrm{mg} / \mathrm{dl}$ for TC (Table 1). $11.4 \%$ had less than or equal 10 years of education and $33.0 \%$ had more than or equal 14 years of education. The median income was 1448.7 Euro/month. The mean number of effect alleles were $78.3 \pm 5.1$ for the $\mathrm{GES}_{\mathrm{HDL}-\mathrm{C}}\left(\mathrm{GES}_{\mathrm{HDL}-\mathrm{C}-\mathrm{EXT}}\right.$ : $\left.136.4 \pm 6.6\right), 55.1 \pm 4.5$ for the $\mathrm{GES}_{\mathrm{LDL}-\mathrm{C}}\left(\mathrm{GES}_{\mathrm{LDL}-\mathrm{C}-\mathrm{EXT}}\right.$ : 93.6 \pm 5.9$)$ and $75.7 \pm 5.2$ for the $\mathrm{GES}_{\mathrm{TC}}\left(\mathrm{GES}_{\mathrm{TC}-\mathrm{EXT}}\right.$ : 126.2 \pm 6.6$) .615$ (13.6\%) participants had diabetes mellitus. Correlation matrix of lipid phenotypes, $\mathrm{GES}_{\text {Lipid }}$, education and income is shown in Supplementary Table S5.

SEP inequalities in HDL-C and LDL-C were found in the study population with worse HDL-C and LDL-C profiles observed in lower income and education groups (Table 2). SEP inequalities in TC were not seen. Participants in the lowest education group ( $\leq 10$ years) had a 4.14 (95\%-CI: $-5.82,-2.47) \mathrm{mg} / \mathrm{dl}$ lower HDL-C, a 4.23 (95\%-CI: 0.37, 8.09) mg/dl higher LDL-C and 3.34 (95\%-CI: -0.81, 7.49) mg/dl higher TC level compared to the participants in the highest education group ( $\geq 14$ years) with similar patterns for income (Table 2). On average, 


\begin{tabular}{|c|c|}
\hline & All $(n=4516)$ \\
\hline Age $(\text { years })^{*}\left[\mathrm{n}_{\text {miss }}=0\right]$ & $59.6 \pm 7.8$ \\
\hline Female Sex ${ }^{\#}$ & $2256(50.0 \%)$ \\
\hline \multicolumn{2}{|l|}{ Serum lipid concentration $(\mathrm{mg} / \mathrm{dl})^{*}$} \\
\hline HDL-C [ $\left.\mathrm{n}_{\text {miss }}=1\right]$ & $58.16 \pm 17.29$ \\
\hline LDL-C [n $\left.\mathrm{n}_{\text {miss }}=14\right]$ & $145.41 \pm 36.21$ \\
\hline $\mathrm{TC}\left[\mathrm{n}_{\mathrm{miss}}=0\right]$ & $229.26 \pm 39.18$ \\
\hline Lipid-lowering medication $\left[\mathrm{n}_{\text {miss }}=289\right]^{\#}$ & $557(13.2 \%)$ \\
\hline \multicolumn{2}{|l|}{ Number of lipid effect alleles $\left(\operatorname{GES}_{\text {Lipid }}\right)^{\varsigma}{ }^{*}$} \\
\hline $\mathrm{GES}_{\text {HDL-C }}\left[\mathrm{n}_{\text {miss }}=0\right]$ & $78.3 \pm 5.1$ \\
\hline $\mathrm{GES}_{\mathrm{LDL}-\mathrm{C}}\left[\mathrm{n}_{\text {miss }}=0\right]$ & $55.1 \pm 4.5$ \\
\hline $\mathrm{GES}_{\mathrm{TC}}\left[\mathrm{n}_{\text {miss }}=0\right]$ & $75.7 \pm 5.2$ \\
\hline \multicolumn{2}{|l|}{ Number of lipid effect alleles $\left(\mathrm{GES}_{\text {Lipid-EXT }}{ }^{+}\right)^{*}$} \\
\hline $\mathrm{GES}_{\text {HDL-C-EXT }}\left[\mathrm{n}_{\text {miss }}=0\right]$ & $136.4 \pm 6.6$ \\
\hline $\mathrm{GES}_{\text {LDL-C-EXT }}\left[\mathrm{n}_{\text {miss }}=0\right]$ & $93.6 \pm 5.9$ \\
\hline $\mathrm{GES}_{\mathrm{TC}-\mathrm{EXT}}\left[\mathrm{n}_{\text {miss }}=0\right]$ & $126.2 \pm 6.6$ \\
\hline \multicolumn{2}{|l|}{ Education (years of training) ${ }^{*}\left[\mathrm{n}_{\text {miss }}=13\right]$} \\
\hline$\leq 10$ & $515(11.4 \%)$ \\
\hline $11-13$ & $2502(55.6 \%)$ \\
\hline$\geq 14$ & $1486(33.0 \%)$ \\
\hline Income $(€ /$ month $) \dagger\left[n_{\text {miss }}=283\right]$ & $1448.7(1107.8-1874.7)$ \\
\hline Body mass index $(\mathrm{kg} / \mathrm{m} 2)^{*}\left[\mathrm{n}_{\text {miss }}=25\right]$ & $27.92 \pm 4.64$ \\
\hline No physical activity ${ }^{\#}$ & $2302(51.0 \%)$ \\
\hline Current smoking ${ }^{\#}\left[\mathrm{n}_{\text {miss }}=7\right]$ & $1069(23.7 \%)$ \\
\hline Alcohol consumption $\dagger$ (g/week) [ $\left.\mathrm{n}_{\text {miss }}=112\right]$ & $13.9(0-64.5)$ \\
\hline Diabetes mellitus $^{\#}$ & $615(13.6 \%)$ \\
\hline
\end{tabular}

Table 1. Characteristics of analysis population. $\mathrm{n}_{\text {miss }}=$ number of participants with missing values, HDL-C = high-density lipoprotein cholesterol, LDL-C = low-density lipoprotein cholesterol, TC = total cholesterol, GES = genetic effect allele sum score, diabetes mellitus is defined as self-reported diabetes mellitus, or fasting blood glucose level $\geq 126 \mathrm{mg} / \mathrm{dl}$ or postprandial blood glucose level $\geq 200 \mathrm{mg} / \mathrm{dl}$ or if the use of anti-diabetic medication was documented. ${ }^{*}$ Mean \pm standard deviation (SD) ${ }^{\#}$ Proportion (\%) ${ }^{\dagger}$ Median (first quartile-third quartile) ${ }^{\S} \mathrm{GES}_{\text {Lipid }}$ based on ${ }^{14+} \mathrm{GES}_{\text {Lipid-EXT }}$ based on ${ }^{14-17}$

a 2.91 (95\%-CI: $(2.45,3.37)) \mathrm{mg} / \mathrm{dl}$ higher HDL-C, a 6.17 (95\%-CI: 5.13, 7.20) mg/dl higher LDL-C and a 7.33 (95\%-CI: 6.24, 8.48) $\mathrm{mg} / \mathrm{dl}$ higher TC were seen per standard deviation of the respective $\mathrm{GES}_{\text {Lipid }}$ (Table 2). The explained variance $\left(\mathrm{R}^{2}\right)$ of $\mathrm{GES}_{\mathrm{HDL}-\mathrm{C}} / \mathrm{GES}_{\mathrm{LDL}-\mathrm{C}} / \mathrm{GES}_{\mathrm{TC}}$ on their respective lipid was 2.9/ 2.9/3.6\%.

In the linear regression analysis including interaction terms, effect size estimates of interaction terms showed stronger indication of $\mathrm{GES}_{\mathrm{Lipid}}$ by low education interaction for LDL-C ( $\beta_{\mathrm{GES} \times \text { Education }}$ : 3.87 ; 95\%-CI: -7.47, -0.32) compared to TC ( $\beta_{\mathrm{GES} \times \text { Education }}$ : $-3.64 ; 95 \%$-CI: $\left.-7.44 ; 0.16\right)$ and HDL-C $\left(\beta_{\mathrm{GES} \times \text { Education }}\right.$ : 0.56 ; $95 \%$-CI: $\left.-2.09 ; 1.02\right)$ using the highest education group as reference (Table 3 ). The negative interaction coefficient showed that in the lower education group genetic effects of $\mathrm{GES}_{\text {Lipid }}$ were less strong. The effect size estimates for the $\mathrm{GES}_{\text {Lipid }}$ by income interaction were directionally consistent, except for TC, but substantially smaller in magnitude.

In the stratified analysis, the two higher education groups compared to the lowest showed stronger genetic effect size estimates per GES $_{\text {Lipid }}$ standard deviation for LDL-C and TC, supporting the results of the interaction analysis (Fig. 1). The results for HDL-C followed the same pattern, but the difference in effect size between the highest and the lowest education group was considerably less strong, while the $95 \%$ confidence interval of the effect in the lowest education group was completely overlapping with the $95 \%$ confidence intervals of both higher education groups. The results of the stratified analysis for income did not follow a clear pattern (Fig. 1). The partial $\mathrm{R}^{2}$ (explained proportion of variance) of the $\mathrm{GES}_{\mathrm{LDL}-\mathrm{C}}$ on LDL-C and GES $\mathrm{TC}_{\mathrm{T}}$ on TC in the respective education groups was higher in the two higher education groups (LDL-C: high education group $\mathrm{R}^{2}=0.033$, middle education group $\mathrm{R}^{2}=0.035$; TC: high education group $\mathrm{R}^{2}=0.044$, middle education group $\mathrm{R}^{2}=0.038$ ) compared to the lower education group (LDL-C: $\mathrm{R}^{2}=0.005$; TC: $\mathrm{R}^{2}=0.013$ ). For Income and HDL-C this trend could not be observed.

The analysis of single reference joint effects for lipids describe the relationship between SEP and GES Lipid $_{\text {. }}$ on lipids in detail by comparing effects of different combinations of SEP groups and $\mathrm{GES}_{\text {Lipid }}$ tertiles. Each beta estimate represent the increase in lipids of the specific group compared to the reference group. Reference group was selected as the combination of $\mathrm{GES}_{\mathrm{LDL}-\mathrm{C}}$ and SEP group representing the lowest CVD risk (equally applied for $\mathrm{GES}_{\mathrm{TC}}$ and $\mathrm{GES}_{\mathrm{HDL}-\mathrm{C}}$ ). For HDL-C beta estimates showed a downward trend between and within education groups with decreasing years of education and decreasing number of effect alleles. Compared to the reference group with the highest education and highest $\mathrm{GES}_{\mathrm{HDL}-\mathrm{C}}$, participants with the lowest education and the lowest 


\begin{tabular}{|c|c|c|c|c|c|c|}
\hline & \multicolumn{2}{|l|}{ HDL-C } & \multicolumn{2}{|l|}{ LDL-C } & \multicolumn{2}{|l|}{ TC } \\
\hline & $\beta(95 \%-C I)$ & $p$ & $\beta(95 \%-C I)$ & $p$ & $\beta(95 \%-C I)$ & $p$ \\
\hline \multicolumn{7}{|c|}{ Lipid $\sim$ Education + age + sex } \\
\hline $\mathrm{n}$ & \multicolumn{2}{|l|}{4502} & \multicolumn{2}{|l|}{4489} & \multicolumn{2}{|l|}{4503} \\
\hline Intercept & $34.28(30.40 ; 38.15)$ & $2.85^{\star} 10^{-65}$ & $129.13(120.22 ; 138.05)$ & $2.3^{\star} 10^{-163}$ & $196.18(186.60 ; 205.76)$ & $1.2^{\star} 10^{-30}$ \\
\hline Age & $0.05(-0.01 ; 0.11)$ & 0.092 & $0.26(0.12 ; 0.39)$ & $2.9^{*} 10^{-04}$ & $0.34(0.19 ; 0.49)$ & $6.3^{\star} 10^{-06}$ \\
\hline Sex & $14.85(13.87 ; 15.82)$ & $5.0^{\star} 10^{-179}$ & $-0.72(-2.96 ; 1.52)$ & 0.530 & $7.75(5.34 ; 10.16)$ & $3.1^{\star} 10^{-10}$ \\
\hline Education (low) & $-4.14(-5.82 ;-2.47)$ & $1.4^{\star} 10^{-06}$ & $4.23(0.37 ; 8.09)$ & 0.032 & $3.34(-0.81 ; 7.49)$ & 0.115 \\
\hline Education & $-1.77(-2.83 ;-0.72)$ & $9.6^{\star} 10^{-04}$ & $2.95(0.53 ; 5.37)$ & 0.017 & $1.21(-1.39 ; 3.81)$ & 0.360 \\
\hline Education (high) & Ref & - & Ref & - & Ref & - \\
\hline \multicolumn{7}{|c|}{ Lipid $\sim$ Income + age + sex } \\
\hline $\mathrm{n}$ & \multicolumn{2}{|l|}{4232} & \multicolumn{2}{|l|}{4220} & \multicolumn{2}{|l|}{4233} \\
\hline Intercept & $36.17(32.24 ; 40.10)$ & $3.1^{\star} 10^{-70}$ & $127.00(118.02 ; 136.00)$ & $1.9^{*} 10^{-155}$ & $194.28(184.68 ; 203.88)$ & $7.6^{\star} 10^{-29}$ \\
\hline Age & $0.03(-0.03 ; 0.09)$ & 0.380 & $0.27(0.13 ; 0.41)$ & $1.9^{\star} 10^{-04}$ & $0.36(0.21 ; 0.51)$ & $2.4^{\star} 10^{-06}$ \\
\hline Sex & $14.11(13.16 ; 15.07)$ & $6.5^{\star} 10^{-169}$ & $0.01(-2.17 ; 2.19)$ & 0.995 & $7.95(5.62 ; 10.29)$ & $2.5^{\star} 10^{-11}$ \\
\hline Income (low) & $-1.09(-2.24 ;-0.05)$ & 0.061 & $3.91(1.29 ; 6.53)$ & $3.4^{\star} 10^{-03}$ & $2.62(-0.17 ; 5.42)$ & 0.066 \\
\hline Income & $-1.55(-2.75 ;-0.34)$ & 0.012 & $2.71(-0.05 ; 5.46)$ & 0.054 & $1.13(-1.82 ; 4.07)$ & 0.452 \\
\hline Income (high) & Ref & - & Ref & - & Ref & - \\
\hline \multicolumn{7}{|c|}{ Lipid $\sim$ GES $_{\text {Lipid }}+$ age + sex } \\
\hline $\mathrm{n}$ & \multicolumn{2}{|l|}{4515} & \multicolumn{2}{|l|}{4502} & \multicolumn{2}{|l|}{4516} \\
\hline Intercept & $-8.42(-16.33 ;-0.51)$ & 0.037 & $53.76(38.51 ; 69.01)$ & $5.6^{\star} 10^{-12}$ & $89.74(71.21 ; 108.26)$ & $3.4^{*} 10^{-21}$ \\
\hline Age & $0.02(-0.04 ; 0.08)$ & 0.533 & $0.27(0.14 ; 0.41)$ & $6.4^{\star} 10^{-05}$ & $0.35(0.21 ; 0.49)$ & $1.8^{\star} 10^{-06}$ \\
\hline Sex & $14.05(13.15 ; 15.00)$ & $6.2^{\star} 10^{-185}$ & $0.06(-2.02 ; 2.14)$ & 0.956 & $8.00(5.77 ; 10.23)$ & $2.2^{\star} 10^{-12}$ \\
\hline GES $_{\text {Lipid }}$ & $2.91(2.45 ; 3.37)$ & $7.5^{\star} 10^{-35}$ & $6.17(5.13 ; 7.20)$ & $7.1^{\star} 10^{-31}$ & $7.33(6.24 ; 8.48)$ & $2.0^{\star} 10^{-37}$ \\
\hline \multicolumn{7}{|c|}{ Lipid $\sim$ GES $_{\text {Lipid }}+$ Education + age + sex } \\
\hline $\mathrm{n}$ & \multicolumn{2}{|l|}{4502} & \multicolumn{2}{|l|}{4489} & \multicolumn{2}{|l|}{4503} \\
\hline Intercept & $-9.92(-17.85 ;-1.98)$ & 0.014 & $54.40(39.06 ; 69.75)$ & $4.2^{\star} 10^{-12}$ & $90.80(72.16 ; 109.44)$ & $2.1^{\star} 10^{-21}$ \\
\hline Age & $0.05(-0.01 ; 0.11)$ & 0.110 & $0.25(0.11 ; 0.38)$ & $4.0^{\star} 10^{-04}$ & $0.33(0.18 ; 0.48)$ & $9.4^{\star} 10^{-06}$ \\
\hline Sex & $14.79(13.83 ; 15.75)$ & $3.0^{\star} 10^{-183}$ & $-1.03(-3.23 ; 1.18)$ & 0.363 & $7.35(4.98 ; 9.71)$ & $1.3^{\star} 10^{-09}$ \\
\hline GES $_{\text {Lipid }}$ & $2.91(2.45 ; 3.36)$ & $5.1^{\star} 10^{-35}$ & $6.17(5.13 ; 7.20)$ & $6.9^{*} 10^{-31}$ & $7.33(6.19 ; 8.42)$ & $4.5^{\star} 10^{-37}$ \\
\hline Education (low) & $-4.12(-5.77 ;-2.47)$ & $1.0^{*} 10^{-06}$ & $4.73(0.92 ; 8.53)$ & 0.015 & $3.52(-0.55 ; 7.60)$ & 0.090 \\
\hline Education & $-1.81(-2.84 ;-0.77)$ & $6.2^{\star} 10^{-04}$ & $3.41(1.03 ; 5.79)$ & $5.1^{\star} 10^{-03}$ & $1.49(-1.06 ; 4.05)$ & 0.252 \\
\hline Education (high) & Ref & - & Ref & - & Ref & - \\
\hline \multicolumn{7}{|c|}{ Lipid $\sim$ GES $_{\text {Lipid }}+$ Income + age + sex } \\
\hline $\mathrm{n}$ & \multicolumn{2}{|l|}{4232} & \multicolumn{2}{|l|}{4220} & \multicolumn{2}{|l|}{4233} \\
\hline Intercept & $-7.34(-15.49 ; 0.82)$ & 0.078 & $56.39(40.66 ; 72.12)$ & $2.4^{\star} 10^{-12}$ & $92.54(73.60 ; 111.48)$ & $1.6^{*} 10^{-21}$ \\
\hline Age & $0.02(-0.04 ; 0.08)$ & 0.480 & $0.27(0.13 ; 0.40)$ & $1.8^{\star} 10^{-04}$ & $0.35(0.20 ; 0.50)$ & $3.0^{\star} 10^{-06}$ \\
\hline Sex & $14.08(13.14 ; 15.01)$ & $4.3^{\star} 10^{-173}$ & $-0.14(-2.30 ; 2.01)$ & 0.895 & $7.67(5.38 ; 9.96)$ & $6.1^{\star} 10^{-11}$ \\
\hline GES $_{\text {Lipid }}$ & $2.86(2.40 ; 3.32)$ & $5.2^{\star} 10^{-32}$ & $5.81(4.73 ; 6.89)$ & $3.5^{\star} 10^{-26}$ & $7.07(5.93 ; 8.22)$ & $2.1^{\star} 10^{-33}$ \\
\hline Income (low) & $-1.08(-2.20 ; 0.05)$ & 0.061 & $3.57(0.98 ; 6.15)$ & $6.8^{\star} 10^{-03}$ & $2.19(-0.56 ; 4.94)$ & 0.119 \\
\hline Income & $-1.49(-2.68 ;-0.31)$ & 0.014 & $2.29(-0.43,5.01)$ & 0.098 & $0.73(-2.17 ; 3.62)$ & 0.622 \\
\hline Income (high) & Ref & & Ref & & Ref & \\
\hline
\end{tabular}

Table 2. Sex- and age- adjusted effects per $\mathrm{GES}_{\text {Lipid }}$ standard deviation and corresponding $95 \%$ confidence intervals (95\% CI) on high-density lipoprotein cholesterol (HDL-C), low-density lipoprotein cholesterol (LDL-C) and total cholesterol (TC) in linear regression models including main effects of education groups ( $\leq 10$ years $/ 11-13$ years/ $\geq 14$ years), income tertiles and genetic effect allele sum scores $\left(\mathrm{GES}_{\text {Lipid }}\right)$ based on ${ }^{14}$.

$\mathrm{GES}_{\text {HDL-C }}$ showed a $9.85 \mathrm{mg} / \mathrm{dl}$ lower HDL-C level. Slightly smaller joint effects were observed for income and $\mathrm{GES}_{\mathrm{HDL}-\mathrm{C}}$ on HDL-C still following the same pattern (Fig. 2). The joint effects for LDL-C and TC showed an upward trend with decreasing years of education and increasing number of effect alleles. Participants with highest CVD-risk (highest GES $_{\text {Lipid }}$ and lowest education) had a $13.36 \mathrm{mg} / \mathrm{dl}$ higher LDL-C and a $15.40 \mathrm{mg} / \mathrm{dl}$ higher TC level than those with the lowest CVD-risk (highest education and lowest $\mathrm{GES}_{\text {Lipid }}$ ). Slightly stronger joint

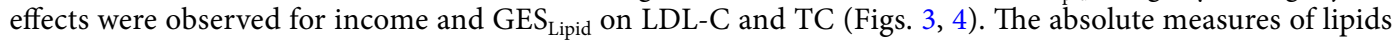
in each of the different combinations of SEP groups and GES $_{\text {Lipid }}$ tertiles show the same pattern as in the single reference joint effect analysis (Supplementary Figures S3-S5). Participants in the highest GES $_{\text {Lipid }}$ tertile and the lowest education had on average $8.8 / 14.0 / 21.1 \mathrm{mg} / \mathrm{dl}$ higher HDL-C/LDL-C/TC level than participants in the 


\begin{tabular}{|c|c|c|c|c|c|c|}
\hline & \multicolumn{2}{|l|}{ HDL-C } & \multicolumn{2}{|l|}{ LDL-C } & \multicolumn{2}{|l|}{ TC } \\
\hline & $\beta(95 \%-C I)$ & $p$ & $\beta(95 \%-C I)$ & $p$ & $\beta(95 \%-C I)$ & $p$ \\
\hline \multicolumn{7}{|c|}{ 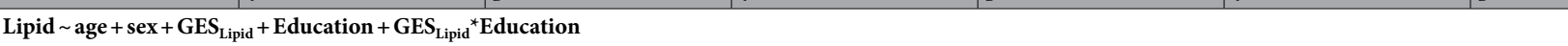 } \\
\hline $\mathrm{n}$ & \multicolumn{2}{|l|}{4502} & \multicolumn{2}{|l|}{4489} & \multicolumn{2}{|l|}{4503} \\
\hline Intercept & $-11.50(-23.95 ; 1.00)$ & 0.070 & $49.89(25.51 ; 74.26)$ & $6.1^{\star} 10^{-05}$ & $80.32(50.25 ; 110.40)$ & $1.7^{\star} 10^{-07}$ \\
\hline Age & $0.05(-0.01 ; 0.11)$ & 0.106 & $0.25(0.11 ; 0.38)$ & $4.1^{\star} 10^{-04}$ & $0.33(0.19 ; 0.478)$ & $8.1^{\star} 10^{-06}$ \\
\hline Sex & $14.79(13.83 ; 15.75)$ & $3.9^{\star} 10^{-183}$ & $-1.07(-3.28 ; 1.14)$ & 0.341 & $7.35(5.00 ; 9.72)$ & $1.2^{\star} 10^{-09}$ \\
\hline $\mathrm{GES}_{\text {Lipid }}$ & $3.01(2.24 ; 3.77)$ & $5.2^{\star} 10^{-14}$ & $6.53(4.68 ; 8.37)$ & $5.2^{\star} 10^{-12}$ & $8.01(6.08 ; 9.98)$ & $1.2^{\star} 10^{-15}$ \\
\hline Education (low) & $4.33(-19.59 ; 28.26)$ & 0.723 & $52.31(8.39 ; 96.24)$ & 0.020 & $56.57(0.93 ; 112.21)$ & 0.046 \\
\hline Education & $-0.68(-16.00 ; 14.64)$ & 0.931 & $1.84(-26.52 ; 30.20)$ & 0.899 & $8.88(-27.09 ; 44.86)$ & 0.628 \\
\hline Education (high) & Ref & - & Ref & - & Ref & - \\
\hline $\mathrm{GES}_{\text {Lipid }} \times \mathrm{Edu}^{\#}$ (low) & $-0.56(-2.09 ; 1.02)$ & 0.487 & $-3.87(-7.47 ;-0.32)$ & 0.033 & $-3.64(-7.44 ; 0.16)$ & 0.062 \\
\hline $\mathrm{GES}_{\text {Lipid }} \times \mathrm{Edu}^{*}$ & $-0.05(-1.07 ; 0.92)$ & 0.885 & $0.14(-2.16 ; 2.43)$ & 0.911 & $-0.52(-2.96 ; 1.98)$ & 0.686 \\
\hline $\mathrm{GES}_{\text {Lipid }} \times \mathrm{Edu}^{*}($ high $)$ & Ref & - & Ref & - & Ref & - \\
\hline \multicolumn{7}{|c|}{ Lipid $\sim$ age + sex + GES $_{\text {Lipid }}+$ Income + GES $_{\text {Lipid }}{ }^{*}$ Income } \\
\hline $\mathrm{n}$ & \multicolumn{2}{|l|}{4232} & \multicolumn{2}{|l|}{4220} & \multicolumn{2}{|l|}{4233} \\
\hline Intercept & $-8.60(-21.34 ; 4.14)$ & 0.186 & $55.82(31.47 ; 80.16)$ & $7.2^{\star} 10^{-06}$ & $88.25(58.43 ; 118.07)$ & $7.0^{*} 10^{-09}$ \\
\hline Age & $0.02(-0.04 ; 0.08)$ & 0.483 & $0.27(0.13 ; 0.40)$ & $1.8^{\star} 10^{-04}$ & $0.35(0.20 ; 0.50)$ & $3.2^{\star} 10^{-06}$ \\
\hline Sex & $14.07(13.14 ; 15.01)$ & $5.5^{\star} 10^{-173}$ & $-0.14(-2.41 ; 1.90)$ & 0.897 & $7.66(5.37 ; 9.95)$ & $6.5^{\star} 10^{-11}$ \\
\hline GES $_{\text {Lipid }}$ & $2.96(2.14 ; 3.72)$ & $5.4^{\star} 10^{-13}$ & $5.85(4.01 ; 7.70)$ & $6.8^{\star} 10^{-10}$ & $7.38(5.46 ; 9.31)$ & $1.2^{\star} 10^{-13}$ \\
\hline Income (low) & $0.80(-16.72 ; 18.33)$ & 0.928 & $6.54(-24.83 ; 37.91)$ & 0.683 & $-4.95(-44.85 ; 34.94)$ & 0.808 \\
\hline Income & $0.56(-17.31 ; 18.42)$ & 0.951 & $0.40(-32.81 ; 33.61)$ & 0.981 & $23.38(-18.03 ; 64.79)$ & 0.269 \\
\hline Income (high) & Ref & - & Ref & - & Ref & - \\
\hline $\mathrm{GES}_{\text {Lipid }} \times \operatorname{Inc}^{\$}$ (low) & $-0.10(-1.28 ; 1.02)$ & 0.833 & $-0.23(-2.79 ; 2.30)$ & 0.852 & $0.47(-2.24 ; 3.22)$ & 0.726 \\
\hline $\mathrm{GES}_{\text {Lipid }} \times \operatorname{Inc}^{\S}$ & $-0.15(-1.28 ; 1.02)$ & 0.822 & $0.14(-2.57 ; 2.84)$ & 0.911 & $-1.56(-4.42 ; 1.30)$ & 0.283 \\
\hline $\mathrm{GES}_{\text {Lipid }} \times \operatorname{Inc}^{\$}($ high $)$ & Ref & - & Ref & - & Ref & - \\
\hline
\end{tabular}

Table 3. Sex- and age- adjusted effects per $\mathrm{GES}_{\text {Lipid }}$ standard deviation and corresponding $95 \%$ confidence intervals (95\% CI) on high-density lipoprotein cholesterol (HDL-C), low-density lipoprotein cholesterol (LDL$\mathrm{C}$ ) and total cholesterol (TC) in linear regression models including main effects of a lipid-associated genetic effect allele sum score $\left(\mathrm{GES}_{\text {Lipid }}\right.$ based on ${ }^{14}$ ), indicators of socioeconomic position (SEP; education groups and income tertiles) and interaction terms of $\mathrm{GES}_{\text {Lipid }}$ and indicators of SEP. ${ }^{\#}$ Education, ${ }^{\$}$ Income.

lowest $\mathrm{GES}_{\text {Lipid }}$ tertiles and the highest education. Almost similar measures have been observed for income and $\mathrm{GES}_{\text {Lipid }}$ tertiles.

After including interaction terms of smoking, BMI, physical activity, alcohol consumption and diabetes

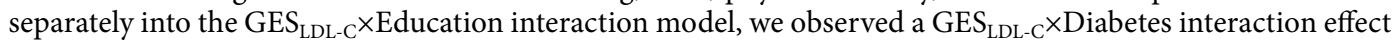
$\left(ß_{\mathrm{GES} \times \text { Diabetes }}:-4.46 ; 95 \%-C I\right.$ : $\left.-7.38,-1.53\right)$ indicating less strong genetic effects on LDL-C in diabetics compared to non-diabetics (Table 4), which was also observed in the stratified analysis (Supplementary Figure S6). Including the $\mathrm{GES}_{\mathrm{LDL}-\mathrm{C}} \times$ Diabetes interaction effect also partly explained the $\mathrm{GES}_{\mathrm{LDL}-\mathrm{C}} \times$ Education interaction effect of the lowest compared to the highest education group, as the effect estimate was attenuated ( $\beta_{\mathrm{GES} \times \mathrm{Education}}:-3.42$; 95\%-CI: $-6.98,0.18$ ) (Table 4). The GES $_{\text {LDL-C }} \times$ Education interaction was not affected by other life style risk factors, as the respective $\mathrm{GES}_{\mathrm{LDL}-\mathrm{C}} \times$ low education interaction effect size estimates did not change in magnitude after including smoking, BMI, physical activity and alcohol consumption in the regression models (Table 4). In addition, results did not indicate $\mathrm{GES}_{\mathrm{LDL}-\mathrm{C}}$ by life style risk factor interactions.

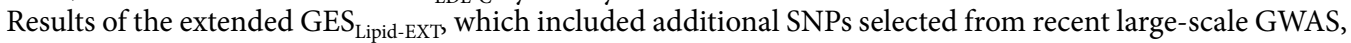
showed overall smaller effect size estimate per GES $_{\text {Lipid-EXT }}$ standard deviation for all three lipid traits compared to the $\mathrm{GES}_{\text {Lipid }}$ (Supplementary Table S6). The explained variance $\left(\mathrm{R}^{2}\right)$ of the extended $\mathrm{GES}_{\text {HDL-C-EXT }}$ was slightly higher $(3.2 \%)$ compared to the $\mathrm{GES}_{\mathrm{HDL}-\mathrm{C}}$ and for the extended $\mathrm{GES}_{\text {LDL-C-EXT }}(2.6 \%)$ and $\mathrm{GES}_{\mathrm{TC}-\mathrm{EXT}}(3.4 \%)$ slightly lower compared to the $\mathrm{GES}_{\mathrm{LDL}-\mathrm{C}}$ and $\mathrm{GES}_{\mathrm{TC}}$. In the extended $\mathrm{GES}_{\mathrm{Lipid}-\mathrm{EXT}}$ by SEP indicator interaction analysis, using the highest SEP groups as reference, effect size estimates of interaction terms were overall slightly smaller in magnitude for all three lipid levels compared to the $\mathrm{GES}_{\text {Lipid }}$ (Supplementary Table S7). Effects of the extended GES $_{\text {Lipid-EXT }}$ stratified by education groups showed similar patterns for HDL-C, LDL-C, and TC compared to the $\mathrm{GES}_{\text {Lipid. }}$. However, differences in the genetic effects between education groups were less strong in magnitude, while for income no difference in the genetic effects were observed (Supplementary Figure S2). The downward trend between and within education groups with decreasing years of education and decreasing number of effect alleles in the analysis of single reference joint effects for HDL-C and the upward trend for LDL-C and TC for both SEP indicators also showed the same pattern using the extended $\mathrm{GES}_{\text {Lipid-EXT }}$ compared to the $\mathrm{GES}_{\text {Lipid }}$ (Supplementary Tables S8-S9).

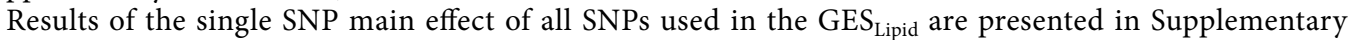
Tables S2-S4. 61 out of 71 HDL-C-, 52 out of 58 LDL-C-, 65 out of 74 TC-associated SNPs were directionally consistent. Single SNP interaction analysis for education and lipid-associated SNPs showed that some SNPs 


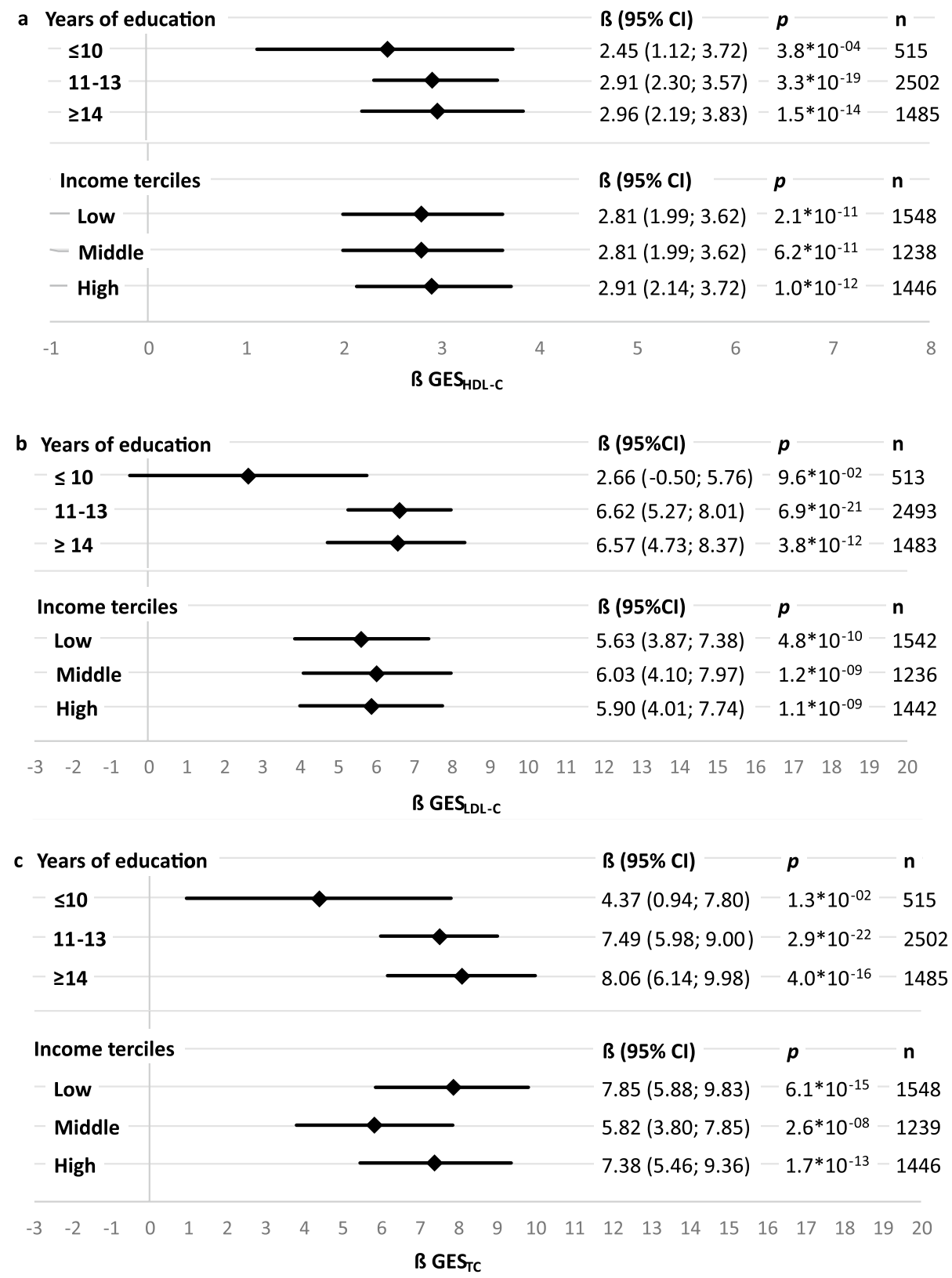

Figure 1. Sex-, age-adjusted effects per GES $_{\text {Lipid }}$ standard deviation and corresponding $95 \%$ confidence interval (95\% CI) of the genetic effect on (a) high-density lipoprotein cholesterol (HDL-C), (b) low-density lipoprotein cholesterol (LDL-C) and (c) total cholesterol (TC), stratified by education groups (years) and income tertiles in linear regression models using the $\mathrm{GES}_{\text {Lipid }}$ based on ${ }^{14}$.

contributed with stronger effects to the observed $\mathrm{GES}_{\mathrm{LDL}-\mathrm{C}}$ by low education interaction on LDL-C in relation to the remaining SNPs (Supplementary Table S10). Similar differences of single SNP interaction effect size estimates were present for HDL-C- and TC-associated SNPs (Supplementary Tables S11-S12). Two of the LDL-Cassociated loci with the strongest indication for interaction with education (i.e., PCSK9, MAFB) were also upon the strongest TC-associated SNPs. The two HDL-C-associated loci with the strongest indication for interaction with education were OR4C46 and $L P L$.

In the sensitivity analysis, main results for all lipid traits did not differ in direction and only slightly in magnitude after adjustment for lipid-lowering medication as well as after the exclusion of participants on lipid-lowering

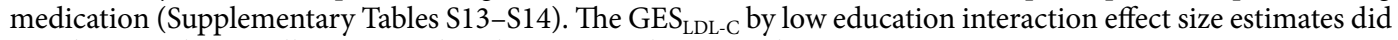
not change substantially compared to the main analysis population. 


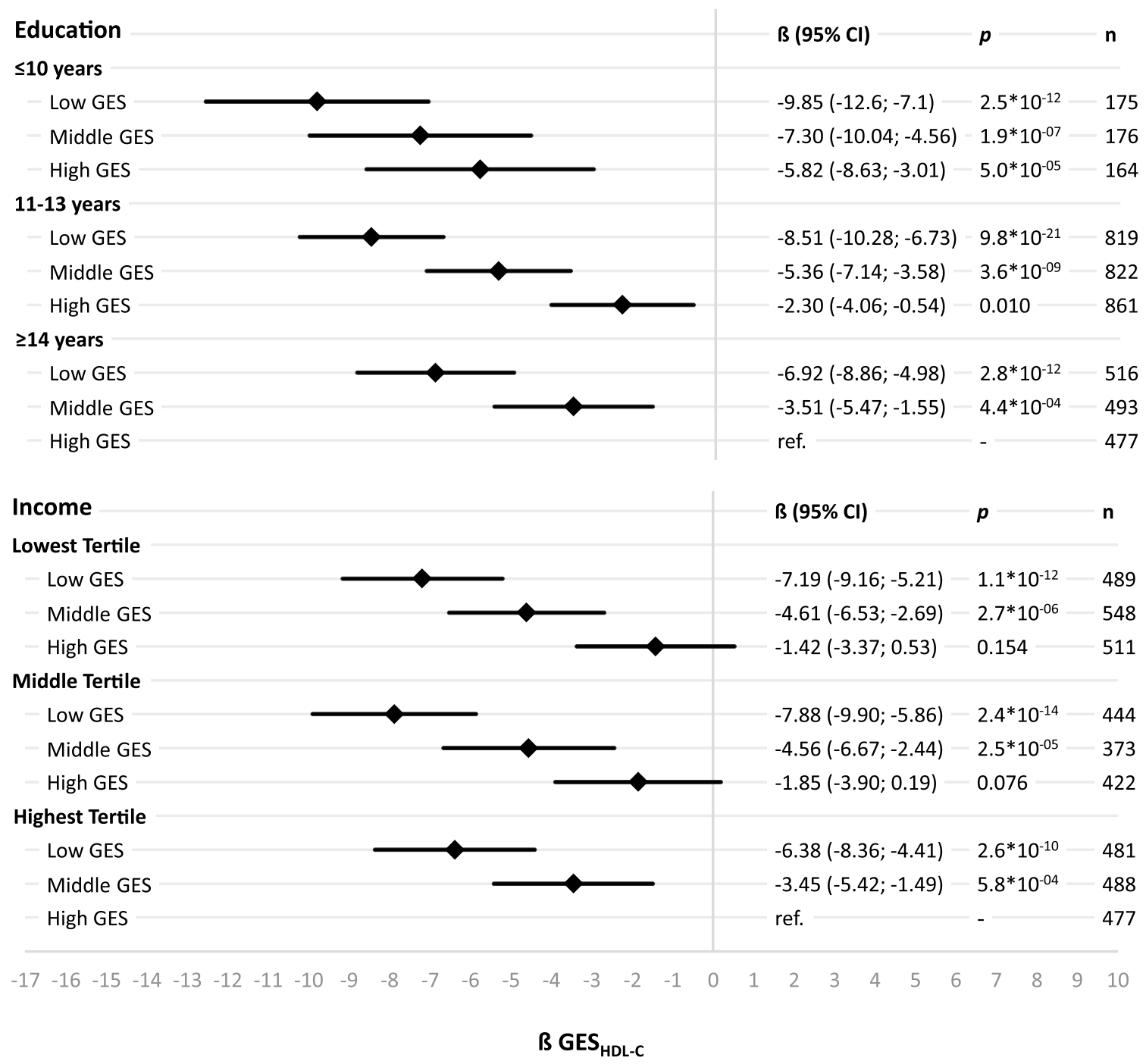

Figure 2. Sex- and age-adjusted effects and corresponding 95\% confidence intervals (95\% CI) on high-density lipoprotein cholesterol (HDL-C) in linear regression models for single reference joint effects of tertiles of a HDL-C-associated genetic effect allele sum score $\left(\mathrm{GES}_{\mathrm{HDL}-\mathrm{C}}\right.$ based on $\left.{ }^{14}\right)$ and socioeconomic position indicators, calculated separately for education groups and income tertiles, with the group of having a high GES $\mathrm{HDL}_{\mathrm{C}}$ and the highest socioeconomic position as reference.

\section{Discussion}

The aim of the study was to investigate whether the SEP indicators education and income interact with genetic sum scores of lipid-increasing effect alleles in a population-based cohort study. To the best of our knowledge, this was the first study investigating $\mathrm{G} \times \mathrm{E}$ interaction with SEP as environmental factor on lipids in adults. Results gave some indication for an interaction between the $\mathrm{GES}_{\mathrm{Lipid}}$ and the SEP indicator education, which was strongest for LDL-C. This was supported by stratified analysis in which the strongest genetic effects on LDL-C were observed in the high education group as well as by single reference joint effect analysis. After including information on smoking, BMI, physical activity, alcohol consumption and diabetes mellitus into analysis, there was an indication that a $\mathrm{GES}_{\mathrm{LDL}-\mathrm{C}}$ by diabetes mellitus interaction partly explained the observed $\mathrm{GES}_{\text {LDL-C }}$ by low education interaction. Using the extended $\mathrm{GES}_{\text {Lipid-EXT }}$ in comparison to the $\mathrm{GES}_{\text {Lipid }}$, effect size measures were smaller but directionally consistent. Li et al. reported an interaction between rs2206734 SNP (CDKAL1), a favorable childhood environment and birthweight on metabolically obese, normal weight phenotype (defined as the presence of hypertension, hypertriglyceridemia, low serum HDL-C or impaired fasting plasma concentrations of glucose) in Chinese children. Their findings suggest that a favorable childhood environment represented by a composite score consisting of parental education, annual household income, high physical activity and fruit consumption can further amplify a protective effect of the CDKAL1 locus in children with a pediatric metabolic syndrome and high birthweight ${ }^{33}$. However, this study investigated a composite environmental score with parental SEP on a composite children's phenotype in Chinese and can therefore only indirectly be compared with present study results. 


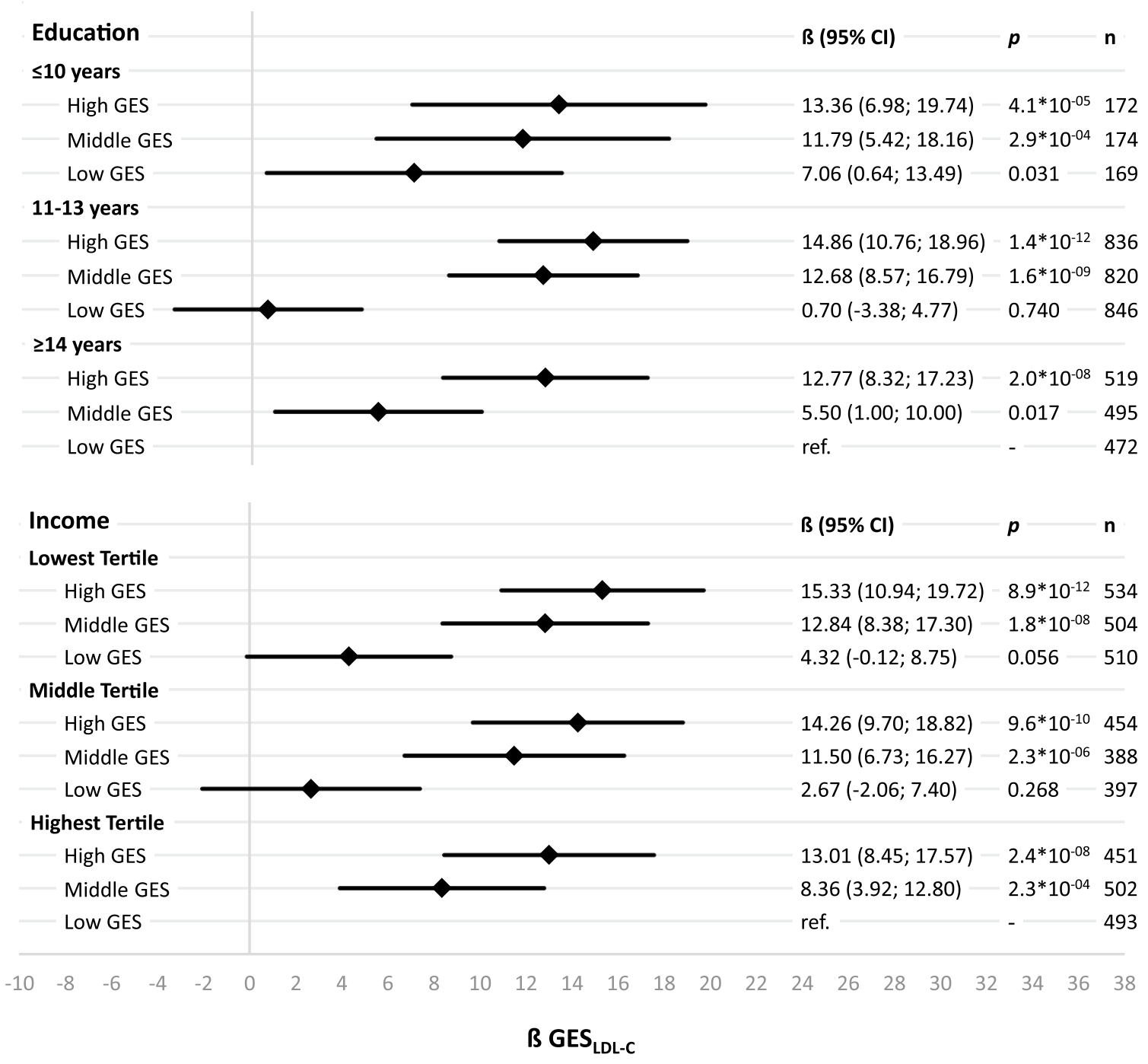

Figure 3. Sex- and age-adjusted effects and corresponding $95 \%$ confidence intervals ( $95 \% \mathrm{CI})$ on low-density lipoprotein cholesterol (LDL-C) in linear regression models for single reference joint effects of tertiles of a LDL-C-associated genetic effect allele sum score $\left(\mathrm{GES}_{\mathrm{LDL}-\mathrm{C}}\right.$ based on $\left.{ }^{14}\right)$ and socioeconomic position indicators, calculated separately for education groups and income tertiles, with the group of having a low GES $\mathrm{LDL}_{\mathrm{C}}$ and the highest socioeconomic position as reference.

Recent studies have investigated statistical interactions between genetic risk scores and SEP-related lifestyle factors or health behaviors such as physical activity, dietary patterns and BMI and their effects on lipids ${ }^{27,28}$. Cole et al. (2014) have demonstrated in a population of European ancestry that the effect of a genetic risk score consisting of HDL-C-increasing alleles has been stronger for lean than for obese (BMI $\left.\geq 35 \mathrm{~kg} / \mathrm{m}^{2}\right)$ study participants. These interactions have been largely driven by the SNPs rs3764261 (CETP), rs4846914 (GALNT2), rs7241918 $(L I P G)$ and rs6065906 (PLTP $)^{28}$. As high education is strongly associated with low BMI ${ }^{48}$, these results may at least partly reflect the results of the present study. However, in the present study the SNPs representing the loci CETP, GALNT2, LIPG and PLTP did not show indication for SNP by education interaction on HDL-C.

Justesen et al. (2015) have reported an interaction between a genetic risk score of HDL-C-decreasing effect alleles and physical activity in a Danish population $(n=5961)$, suggesting that the genetic risk score has exerted a smaller effect in physically active compared to inactive individuals. However, this interaction was statistically not significant in a replication cohort of smaller sample size ${ }^{27}$. As higher education is usually associated with a higher level of physical activity ${ }^{49}$, the results of the present study may represent the same interaction signal, i.e. a stronger genetic effect of HDL-C-increasing effect alleles on HDL-C in population groups of higher education groups.

Recent $\mathrm{SNP} \times \mathrm{E}$ interaction analyses have identified several lipid-associated loci interacting with lifestyle factors such as smoking and diet ${ }^{50-52}$. While Junyent et al. (2009) have reported an interaction of rs6720173 (ABCG5) and rs11887534, rs6709904, rs4148217 (ABCG8) with smoking ${ }^{50}$, Lu et al. (2010) have shown an interaction of rs174546 (FADS1) with intake of n-3 and n-6 polyunsaturated fatty acid ${ }^{51}$ and Kim et al. (2013) have demonstrated an interaction of rs2072183 (NPC1L1) with cholesterol intake in male only ${ }^{52}$. Although SEP is strongly associated with smoking and dietary factors, none of these loci showed indication for interaction with education in the present study. The $\mathrm{GES}_{\text {LDL-C }} \times$ Diabetes mellitus interaction on LDL-C observed in the present 


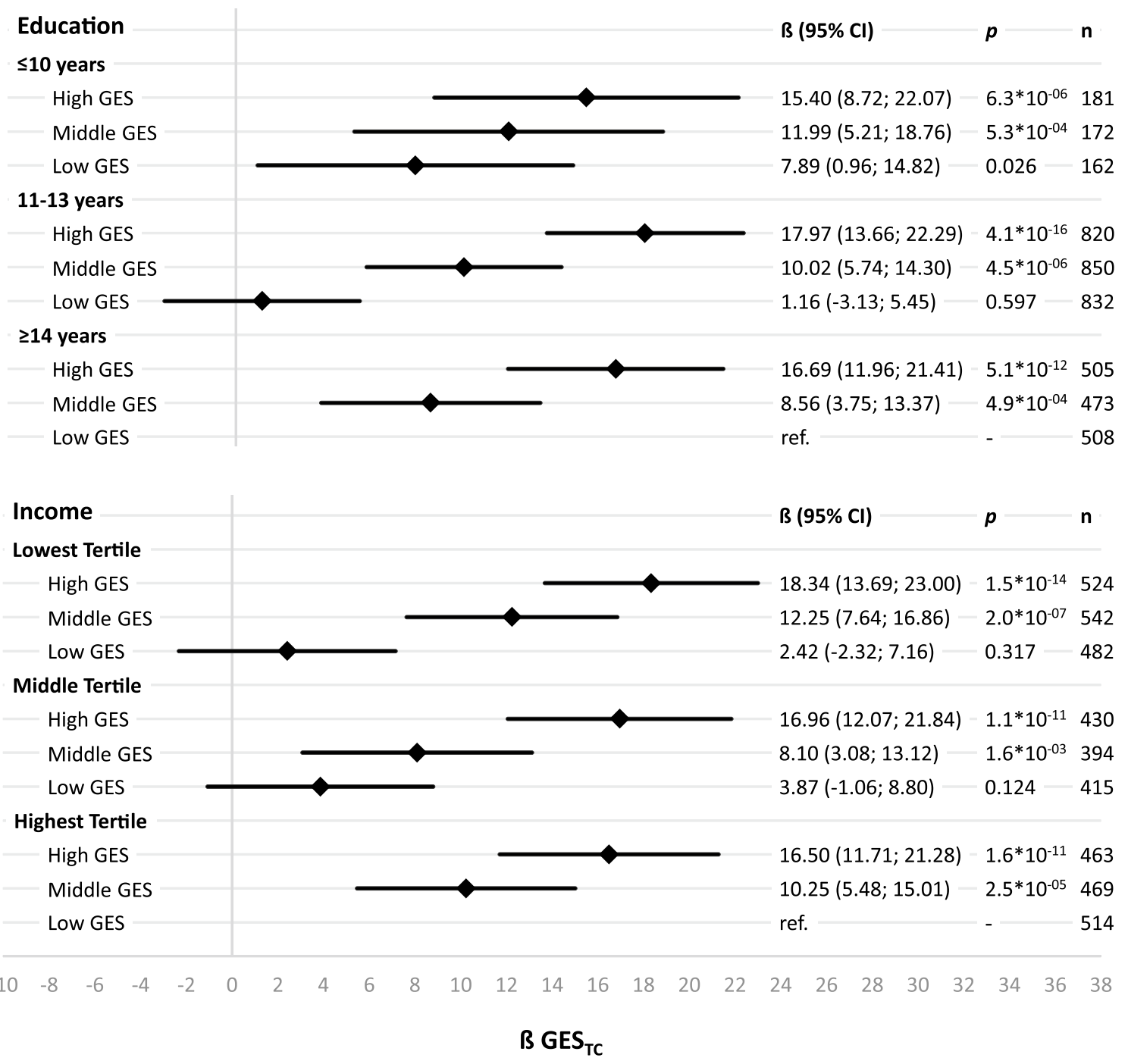

Figure 4. Sex- and age-adjusted effects and corresponding 95\% confidence intervals (95\% CI) on total cholesterol (TC) in linear regression models for single reference joint effects of tertiles of a TC-associated genetic effect allele sum score $\left(\mathrm{GES}_{\mathrm{TC}}\right.$ based on $\left.^{14}\right)$ and socioeconomic position indicators, calculated separately for education groups and income tertiles, with the group of having a low $\mathrm{GES}_{\mathrm{TC}}$ and the highest socioeconomic position as reference.

study partly explained the $\mathrm{GES}_{\mathrm{LDL}-\mathrm{C}} \times$ Education interaction. Deng et al. found a Gene $\times$ Diabetes mellitus interaction. In their study the SNP rs16996148 (CILP2) decreased the risk of hyperlipidemia, whereas rs16996148 GT/ TT and diabetes mellitus as well as rs $16996148 \mathrm{TT}$ and diabetes mellitus increased the risk of hyperlipidemia ${ }^{29}$.

In the present analysis, smaller effects on lipids were observed for using the extended $\mathrm{GES}_{\text {Lipid-EXT }}$ compared to the $\mathrm{GES}_{\text {Lipid }}$. This may be caused by the overall smaller effect size of newly discovered loci as a result of larger analysis populations in recent GWAS, making it possible to detect risk alleles with very small effects. It may also be due to the recently published GWAS meta-analyses that were based on single large cohorts potentially producing less generalizable study results ${ }^{16,17}$. The overall smaller main effects of the additional SNPs included in the extended $\mathrm{GES}_{\text {Lipid-EXT }}$ have led to smaller interaction effect size estimates, i.e. less strong impact of SEP on the expression of the average genetic effect of all SNPs included.

It was assumed in the present analysis that the $\mathrm{GES}_{\text {Lipid }}$ represent cumulative causal factors for lipids even if it is most likely that the SNPs used to construct the $\mathrm{GES}_{\text {Lipid }}$ are proxy markers in high LD with the causal genetic variants ${ }^{53}$. The effect of SEP, especially education, on lipids and CVD risk in general was also assumed to be causal, as supported by numerous studies ${ }^{54-56}$ including mendelian randomization studies exploring the association of instrumental variables with CVD and CVD risk factors by using genetic risk scores related to educational attainment ${ }^{57,58}$. However, SEP has no direct causal effect on CVD risk, but is mediated by a complex interplay of social inequalities in risk factors, e.g., access to preventive interventions, lifestyle factors, physiological stress, psychosocial risks, as well as in protective factors ${ }^{54,57,59}$. Results of the present study suggest that SEP may also have an effect on CVD risk by affecting the expression of LDL-C-related genetic risks. One possible mechanism that has been hypothesized in this regard is epigenetic modification. In contrast to an individual's 


\begin{tabular}{|c|c|c|c|c|c|c|c|c|c|c|}
\hline \multirow[b]{2}{*}{ Education } & \multicolumn{2}{|c|}{ 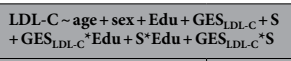 } & \multicolumn{2}{|c|}{ 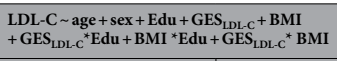 } & \multicolumn{2}{|c|}{ 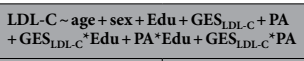 } & \multicolumn{2}{|c|}{ 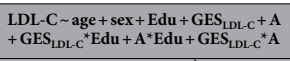 } & \multicolumn{2}{|c|}{ 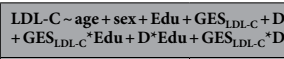 } \\
\hline & $\beta(95 \%-\mathrm{CI})$ & $p$ & $\beta(95 \%-\mathrm{CI})$ & $p$ & $\beta(95 \%-\mathrm{CI})$ & $p$ & $\beta(95 \%-\mathrm{CI})$ & $p$ & $\beta(95 \%-C I)$ & $p$ \\
\hline $\mathrm{n}$ & \multicolumn{2}{|l|}{4489} & \multicolumn{2}{|l|}{4466} & \multicolumn{2}{|l|}{4489} & \multicolumn{2}{|l|}{4382} & \multicolumn{2}{|l|}{4489} \\
\hline Intercept & $41.87(16.61 ; 67.14)$ & $1.2^{*} 10^{-03}$ & $-8.24(-87.26 ; 70.77)$ & 0.840 & $49.58(23.44 ; 75.71)$ & $2.0^{*} 10^{-04}$ & $50.37(23.74 ; 77.00)$ & $2.1^{*} 10^{-04}$ & $42.00(17.20 ; 66.79)$ & $9.1 * 10^{-04}$ \\
\hline Age & \begin{tabular}{|l|}
$0.31(0.17 ; 0.45)$ \\
\end{tabular} & $1.1^{*} 10^{-05}$ & \begin{tabular}{|l|}
$0.21(0.07 ; 0.35)$ \\
\end{tabular} & $2.7^{*} 10^{-03}$ & $0.24(0.11 ; 0.38)$ & $4.9^{*} 10^{-04}$ & $0.25(0.12 ; 0.39)$ & $3.1^{*} 10^{-04}$ & $0.28(0.14 ; 0.42)$ & $5.4^{*} 10^{-05}$ \\
\hline Sex & $-0.75(-2.96 ; 1.47)$ & 0.510 & $-0.36(-2.59 ; 1.86)$ & 0.750 & $-0.83(-3.06 ; 1.39)$ & 0.460 & $-1.33(-3.70 ; 1.04)$ & 0.270 & $-1.67(-3.89 ; 0.55)$ & 0.140 \\
\hline Edu" (low) & $54.93(11.06 ; 98.81)$ & 0.014 & $59.72(11.21 ; 108.22)$ & 0.016 & $54.62(9.89 ; 99.34)$ & 0.017 & $53.68(8.38 ; 98.98)$ & 0.020 & $47.41(3.53 ; 91.30)$ & 0.034 \\
\hline Edu $^{*}$ & $4.06(-24.37 ; 32.49)$ & 0.780 & $9.15(-22.63 ; 40.94)$ & 0.570 & $2.29(-26.48 ; 31.05)$ & 0.880 & $2.94(-25.90 ; 31.79)$ & 0.840 & $0.92(-27.38 ; 29.21)$ & 0.949 \\
\hline Edu* (high) & ref & - & ref & - & ref & - & ref & - & ref & - \\
\hline GES $_{\text {LDL-C }}$ & $6.71(4.82 ; 8.64)$ & $7.1^{*} 10^{-12}$ & $9.41(3.06 ; 15.75)$ & $3.8^{*} 10^{-03}$ & $6.48(4.50 ; 8.51)$ & $2.1^{*} 10^{-10}$ & $6.48(4.41 ; 8.51)$ & $5.7^{*} 10^{-10}$ & $7.16(5.27 ; 9.05)$ & $1.4^{*} 10^{-13}$ \\
\hline S & $9.85(-20.47 ; 40.16)$ & 0.520 & - & - & - & - & - & - & - & - \\
\hline BMI & - & & $2.19(-0.59 ; 4.97)$ & 0.120 & - & - & - & - & - & - \\
\hline $\mathrm{PA}$ & - & & - & - & $-0.04(-26.25 ; 26.16)$ & 1.000 & - & - & - & - \\
\hline A & - & & - & - & - & - & $0.00(-0.13 ; 0.14)$ & 0.950 & - & - \\
\hline $\mathrm{D}$ & & & & & & & & & $46.97(10.46 ; 83.48)$ & 0.012 \\
\hline $\begin{array}{l}\mathrm{GES}_{\text {IDLCC }} \times \\
\text { Edü }^{*} \text { (low) }\end{array}$ & $-4.23(-7.83 ;-0.68)$ & 0.020 & $-3.83(-7.43 ;-0.18)$ & 0.040 & $-3.96(-7.56 ;-0.32)$ & 0.033 & $-3.87(-7.56 ;-0.18)$ & 0.040 & $-3.42(-6.98 ; 0.18)$ & 0.061 \\
\hline $\begin{array}{l}\mathrm{GES}_{\mathrm{LDL}-\mathrm{C}} \times \\
\mathrm{Edu}^{\text {D }}\end{array}$ & $0.00(-2.30 ; 2.30)$ & 1.000 & $0.14(-2.16 ; 2.48)$ & 0.900 & $0.05(-2.30 ; 2.39)$ & 0.970 & $0.09(-2.25 ; 2.43)$ & 0.950 & $0.223(-2.07 ; 2.52)$ & 0.861 \\
\hline $\begin{array}{l}\text { GES }_{\text {LDLC }} \times \\
\text { Edu }^{*} \text { (high) }\end{array}$ & ref & - & ref & - & ref & - & ref & - & ref & - \\
\hline $\mathrm{GES}_{\mathrm{LDL}-\mathrm{C}} \times \mathrm{S}$ & $-0.26(2.70 ; 2.21)$ & 0.850 & - & - & - & - & - & - & - & - \\
\hline $\begin{array}{l}\mathrm{GES}_{\mathrm{LDL}-\mathrm{C}} \times \\
\mathrm{BMI}\end{array}$ & - & - & $-0.09(-0.32 ; 0.14)$ & 0.350 & - & - & - & - & - & - \\
\hline $\begin{array}{l}\mathrm{GES}_{\text {LDLCC }} \\
\times \mathrm{PA}\end{array}$ & - & - & - & - & $0.14(-1.98 ; 2.25)$ & 0.900 & - & - & - & - \\
\hline $\mathrm{GES}_{\mathrm{LDL}-\mathrm{C}} \times \mathrm{A}$ & - & - & - & - & - & - & $0.00(0.00 ; 0.00)$ & 0.960 & - & - \\
\hline $\mathrm{GES}_{\mathrm{LDL}-\mathrm{C}} \times \mathrm{D}$ & & & & & & & & & $-4.46(-7.38 ;-1.53)$ & $3.0^{*} 10^{-03}$ \\
\hline $\begin{array}{l}\mathrm{S} \times \mathrm{Edu}^{*} \\
(\text { low) }\end{array}$ & $6.78(-2.07 ; 15.62)$ & 0.130 & - & - & - & - & - & - & - & - \\
\hline $\mathrm{S} \times \mathrm{Edu}^{*}$ & $-4.51(-10.02 ; 0.99)$ & 0.110 & - & - & - & - & - & - & - & - \\
\hline $\begin{array}{l}\text { S } \times \text { Edu }^{*} \\
(\text { high) }\end{array}$ & ref & - & - & - & - & - & - & - & - & - \\
\hline $\begin{array}{l}\mathrm{BMI} \times \mathrm{Edu}^{*} \\
\text { (low) }\end{array}$ & - & - & $-0.34(-1.06 ; 0.38)$ & 0.350 & - & - & - & - & - & - \\
\hline $\mathrm{BMI} \times \mathrm{Edu}^{2}$ & - & - & $-0.30(-0.82 ; 0.22)$ & 0.260 & - & - & - & - & - & - \\
\hline $\begin{array}{l}\mathrm{BMI} \times \mathrm{Edu}^{*} \\
\text { (high) }\end{array}$ & - & - & ref & - & - & - & - & - & - & - \\
\hline $\begin{array}{l}\mathrm{PA} \times \mathrm{Edu}^{*} \\
\text { (low) }\end{array}$ & - & - & - & - & $-3.33(-10.70 ; 4.04)$ & 0.380 & - & - & - & - \\
\hline $\mathrm{PA} \times \mathrm{Edu}^{*}$ & - & - & - & - & $0.83(-3.82 ; 5.48)$ & 0.730 & - & - & - & - \\
\hline $\begin{array}{l}\mathrm{PA} \times \mathrm{Edu}^{*} \\
\text { (high) }\end{array}$ & - & - & - & - & ref & - & - & - & - & - \\
\hline $\begin{array}{l}\mathrm{A} \times \mathrm{Edu}^{*} \\
\text { (low) }\end{array}$ & - & - & - & - & - & - & $-0.04(-0.08 ; 0.00)$ & 0.057 & - & - \\
\hline $\mathrm{A} \times \mathrm{Edu}^{*}$ & - & - & - & - & - & - & $0.00(-0.02 ; 0.02)$ & 0.950 & - & - \\
\hline $\begin{array}{l}\mathrm{A} \times \mathrm{Edu}^{*} \\
\text { (high) }\end{array}$ & - & - & - & - & - & - & ref & - & - & - \\
\hline $\begin{array}{l}\mathrm{D} \times \mathrm{Edu}^{*} \\
\text { (low) }\end{array}$ & & & & & & & & & $-0.36(-9.95 ; 9.23)$ & 0.941 \\
\hline $\mathrm{D} \times \mathrm{Edu}^{*}$ & & & & & & & & & $1.52(-5.31 ; 8.34)$ & 0.663 \\
\hline $\begin{array}{l}\mathrm{D} \times \mathrm{Eddu}^{*} \\
\text { (high) }\end{array}$ & & & & & & & & & ref & - \\
\hline
\end{tabular}

Table 4. Sex- and age- adjusted effects per $\mathrm{GES}_{\mathrm{LDL}-\mathrm{C}}$ standard deviation and corresponding $95 \%$ confidence intervals $(95 \% \mathrm{CI})$ on LDL-C in linear regression models including main effects and interaction terms of a LDL-C-associated genetic effect allele score $\left(\mathrm{GES}_{\text {LDL-C }}\right.$ based on $\left.{ }^{14}\right)$, education groups and SEP-related life style risk factors (i.e., current smoking [S], BMI, physical activity [PA], alcohol consumption [A; per $100 \mathrm{~g} /$ week]) and diabetes mellitus [D]. ${ }^{\#}$ Education

genome the epigenome is subject to environmentally induced changes during the life course, but is crucial for the regulation of gene expression. Several studies have indeed reported SEP-related differences in epigenetic markers $^{60,61}$. Interestingly, the lifestyle factors BMI, physical activity, smoking and alcohol consumption did not account for the observed $\mathrm{GES}_{\mathrm{LDL}-\mathrm{C}}$ by education interaction, while diabetes mellitus accounted for it only partly. Consequently, it has to be assumed that other risk factors besides those included in the present analysis may have a mediating effect on the found $\mathrm{GES}_{\mathrm{LDL}-\mathrm{C}}$ by education interaction. One explanation for the stronger genetic effects on LDL-C in higher education groups may be that non-genetic health risks being of lower prevalence in high education groups leading to LDL-C profiles that are stronger affected by genetic than by non-genetic risk factors. This hypothesis is supported by the explained proportion of the variance $\left(\mathrm{R}^{2}\right)$ of the GES $\mathrm{LDL}_{\text {-C }}$ on LDL-C, which was higher in the two higher education groups compared to the lower education group.

The effect of SEP indicators on health is outcome specific and each indicator operates via different pathways linking social factors to health outcomes ${ }^{43,62}$. Even though educational attainment and income are moderately 
correlated $(r=0.45)$ in the present study, the different strength of genetic effect in education groups on LDL-C could not be seen in income tertiles. The net effect of education is reflected among others in the ability to turn health related information into behavior and facilitates understanding of therapeutic measures ${ }^{43}$. Which could support the hypothesis that in highly educated, due to the ability to create environments with less health risks, genetic influence on LDL-C might be stronger. Furthermore, education as a marker of childhood social environment could, due to the duration of exposure until adulthood, be more likely support epigenetic changes. Material resources do not seem to modify genetic risk on LDL-C.

Strengths of the present study were its population-based study sample and the use of two different individual SEP indicators in the analysis. Even though education and income are correlated SEP indicators, each of them represents certain aspects of SEP related to different health behaviors and risks. Moreover, two different $\mathrm{GES}_{\text {Lipid }}$ for each lipid trait were compared, allowing to check for differences in the genetic effects and $\mathrm{G} \times \mathrm{E}$ interactions between scores derived by different GWAS study populations. The sample size and the limited statistical power for single SNP analysis had to be mentioned as limitation of the present study. However, indication for interaction was based on the cumulative genetic risk of the study participants. Another limitation was the cross-sectional design of the study that does not allow for strong conclusions on causality of effects. However, educational attainment is usually acquired in adolescence or early adulthood and lipids were assessed at an older age in the present study. Due to this exposure-outcome temporality reverse causation is very unlikely. Even if the effect of education on lipids was not causal, a modification of the $\mathrm{GES}_{\text {LDL-C }}$ effect on LDL-C by education would still be of interest, because the knowledge of the heterogeneous genetic effects in different education groups could be interesting for CVD risk prediction and genotype-based targeted interventions ${ }^{35}$. Furthermore, this knowledge supports CVD lifestyle-based interventions in lower education groups due to lower genetic effect on LDL-C in these groups. Finally, it cannot be excluded that the indication for a $\mathrm{GES}_{\text {LDL-C }} \times$ Education interaction could have been observed randomly due to the number of independent tests performed. However, the number of independent tests performed are justified through the three lipid end points and the two SEP indicators and we have calculated $95 \%$ confidence intervals to report the precision of the obtained effect size estimates. Furthermore, the interaction analyses results are supported by the results of the stratified and single reference joint effect analysis, which showed a constant pattern across phenotypes and indicated difference in genetic effect sizes between the education groups.

The results of the present study gave some indication for interaction between genetic variants associated with LDL-C and education in a population-based cohort study. Stronger genetic effects were observed in groups of higher education, which seemed to be partly mediated by diabetes mellitus but not by other life style risk factors such as BMI, smoking, physical activity and alcohol consumption. This gave supporting evidence that SEP has an impact on the expression of genetic susceptibility related to LDL-C. Further research is needed to replicate our findings in independent study samples, investigate possible biological mechanisms behind the interaction and to assess the potential of the found gene by SEP interactions for improving CVD prediction. Additionally, our study included only individuals of European origin and therefore the results may not be applied to populations of other ethnicities.

\section{Data availability}

Due to data security reasons (i.e., data contain potentially participant identifying information), the Heinz Nixdorf Recall Study does not allow sharing data as a public use file. However, other authors are allowed to access data upon request, which is the same way authors of the present paper obtained the data. Data requests can be addressed to: recall@uk-essen.de.

Received: 5 October 2020; Accepted: 2 August 2021

Published online: 16 August 2021

\section{References}

1. Wadhera, R. K. et al. A review of low-density lipoprotein cholesterol, treatment strategies, and its impact on cardiovascular disease morbidity and mortality. J Clin Lipidol 10, 472-489 (2016).

2. Piepoli, M. F. et al. 2016 European Guidelines on cardiovascular disease prevention in clinical practice: the Sixth Joint Task Force of the European Society of Cardiology and Other Societies on Cardiovascular Disease Prevention in Clinical Practice (constituted by representatives of 10 societies and by invited experts) developed with the special contribution of the European Association for Cardiovascular Prevention \& Rehabilitation (EACPR). Eur Heart J 37, 2315-2381 (2016).

3. Holmes, M. V. et al. Mendelian randomization of blood lipids for coronary heart disease. Eur Heart J 36, 539-550 (2015).

4. Chapman, M. J. et al. Triglyceride-rich lipoproteins and high-density lipoprotein cholesterol in patients at high risk of cardiovascular disease: evidence and guidance for management. Eur Heart J 32, 1345-1361 (2011).

5. Rohatgi, A. et al. HDL in the 21st Century: a multifunctional roadmap for future HDL research. Circulation 143, 2293-2309 (2021).

6. Sing, C. F., Stengård, J. H. \& Kardia, S. L. R. Genes, environment, and cardiovascular disease. Arterioscler Thromb Vasc Biol 23, 1190-1196 (2003).

7. Luepker, R. V. et al. Socioeconomic status and coronary heart disease risk factor trends. the Minnesota Heart Survey. Circulation 88, 2172-2179 (1993).

8. Perova, N. V. et al. Multi-country comparison of plasma lipid relationship to years of schooling in men and women. Int J Epidemiol 30, 371-379 (2001).

9. Fukuda, Y. \& Hiyoshi, A. Associations of household expenditure and marital status with cardiovascular risk factors in Japanese adults: analysis of nationally representative surveys. J Epidemiol 23, 21-27 (2013).

10. Shohaimi, S. et al. Distribution of lipid parameters according to different socio-economic indicators- the EPIC-Norfolk prospective population study. BMC Public Health 14, 782 (2014).

11. Fujiyoshi, N. et al. Associations between socioeconomic status and the prevalence and treatment of hypercholesterolemia in a general Japanese population: NIPPON DATA2010. J Atheroscler Thromb 25, 606-620 (2018).

12. Benetou, V. et al. Total cholesterol and HDL-cholesterol in relation to socioeconomic status in a sample of 11,645 Greek adults: the EPIC study in Greece. European Prospective Investigation into Nutrition and Cancer. Scand J Public Health 28, 260-265 (2000). 
13. Bruggisser, M. et al. Multivariable analysis of total cholesterol levels in male Swiss Armed Forces conscripts 2006-2012 ( $\mathrm{N}=174,872)$. BMC Cardiovasc Disord 16. https://doi.org/10.1186/s12872-016-0218-2 (2016).

14. Willer, C. J. et al. Discovery and refinement of loci associated with lipid levels. Nat Genet 45, 1274-1283 (2013).

15. Surakka, I. et al. The impact of low-frequency and rare variants on lipid levels. Nat Genet 47, 589-597 (2015).

16. Klarin, D. et al. Genetics of blood lipids among 300,000 multi-ethnic participants of the Million Veteran Program. Nat Genet 50, 1514-1523 (2018)

17. Hoffmann, T. J. et al. A large electronic-health-record-based genome-wide study of serum lipids. Nat Genet 50, 401-413 (2018).

18. Namboodiri, K. K. et al. The Collaborative Lipid Research Clinics Family Study: biological and cultural determinants of familial resemblance for plasma lipids and lipoproteins. Genet Epidemiol 2, 227-254 (1985).

19. Kim, Y. et al. On the estimation of heritability with family-based and population-based samples. BioMed Res Int 2015, 671349 (2015).

20. Winham, S. J. \& Biernacka, J. M. Gene-environment interactions in genome-wide association studies: current approaches and new directions. J Child Psychol Psychiatry 54, 1120-1134 (2013).

21. Manolio, T. A. et al. Finding the missing heritability of complex diseases. Nature 461, 747-753 (2009).

22. Ordovás, J. M., Robertson, R. \& Cléirigh, E. N. Gene-gene and gene-environment interactions defining lipid-related traits. Curr Opin Lipidol 22, 129-136 (2011).

23. Cole, C. B., Nikpay, M. \& McPherson, R. Gene-environment interaction in dyslipidemia. Curr Opin Lipidol 26, 133-138 (2015).

24. Bentley, A. R. et al. Multi-ancestry genome-wide gene-smoking interaction study of 387,272 individuals identifies new loci associated with serum lipids. Nat Genet 51, 636-648 (2019).

25. Kilpeläinen, T. O. et al. Multi-ancestry study of blood lipid levels identifies four loci interacting with physical activity. Nat Commun 10, 376 (2019).

26. Noordam, R. et al. Multi-ancestry sleep-by-SNP interaction analysis in 126,926 individuals reveals lipid loci stratified by sleep duration. Nat Commun 10, 5121 (2019)

27. Justesen, J. M. et al. Interactions of Lipid genetic risk scores with estimates of metabolic health in a Danish population. Circ Cardiovasc Genet 8, 465-472 (2015).

28. Cole, C. B. et al. Adiposity significantly modifies genetic risk for dyslipidemia. J Lipid Res 55, 2416-2422 (2014).

29. Deng, G.-X. et al. Association of the NCAN-TM6SF2-CILP2-PBX4-SUGP1-MAU2 SNPs and gene-gene and gene-environment interactions with serum lipid levels. Aging 12, 11893-11913 (2020).

30. Johnson, W. \& Krueger, R. F. Genetic effects on physical health: lower at higher income levels. Behav Genet 35, 579-590 (2005).

31. Johnson, W. et al. Education reduces the effects of genetic susceptibilities to poor physical health. Int J Epidemiol 39, 406-414 (2010).

32. Frank, M. et al. A genetic sum score of risk alleles associated with body mass index interacts with socioeconomic position in the Heinz Nixdorf Recall Study. PLoS ONE 14. https://doi.org/10.1371/journal.pone.0221252 (2019).

33. Li, G. et al. Interaction between early environment and genetic predisposition instigates the metabolically obese, normal weight phenotype in children: findings from the BCAMS study. Eur J Endocrinol 182, 393-403 (2020).

34. Schmidt, B. et al. Socioeconomic Status Interacts with the Genetic Effect of a Chromosome 9p21.3 Common Variant to Influence Coronary Artery Calcification and Incident Coronary Events in the Heinz Nixdorf Recall Study (Risk Factors, Evaluation of Coronary Calcium, and Lifestyle). Circ Cardiovasc Genet 10. https://doi.org/10.1161/CIRCGENETICS.116.001441 (2017).

35. Pollin, T. I. et al. Genetic modulation of lipid profiles following lifestyle modification or metformin treatment: the Diabetes Prevention Program. PLoS Genet 8, e1002895 (2012).

36. Schmermund, A. et al. Assessment of clinically silent atherosclerotic disease and established and novel risk factors for predicting myocardial infarction and cardiac death in healthy middle-aged subjects: rationale and design of the Heinz Nixdorf RECALL Study. Risk Factors, Evaluation of Coronary Calcium and Lifestyle. Am Heart J 144, 212-218 (2002).

37. Stang, A. et al. Baseline recruitment and analyses of nonresponse of the Heinz Nixdorf Recall Study: identifiability of phone numbers as the major determinant of response. Eur J Epidemiol 20, 489-496 (2005).

38. Hoffmann, W. et al. Guidelines and recommendations for ensuring Good Epidemiological Practice (GEP): a guideline developed by the German Society for Epidemiology. Eur J Epidemiol 34, 301-317 (2019).

39. Erbel, R. et al. Gender-specific association of coronary artery calcium and lipoprotein parameters: the Heinz Nixdorf Recall Study. Atherosclerosis 229, 531-540 (2013).

40. UNESCO. International Standard Classification of Education ISCED 1997, http://uis.unesco.org/sites/default/files/documents/inter national-standard-classification-of-education-1997-en_0.pdf.

41. Asghar Zaidi M, Hagenaars AJM, de Voss K. Poverty statistics in the late 1980s: Research base on micro-date., https://op.europa. eu/en/publication-detail/-/publication/9c787f17-acb6-4f4b-badc-49a2310e65f7 (1995, accessed 13 March 2020).

42. Galobardes, B. et al. Indicators of socioeconomic position (part 1). J Epidemiol Community Health 60, 7-12 (2006).

43. Geyer, S. et al. Education, income, and occupational class cannot be used interchangeably in social epidemiology. Empirical evidence against a common practice. J Epidemiol Community Health 60, 804-810 (2006).

44. Howie BN, Donnelly P, Marchini J. A flexible and accurate genotype imputation method for the next generation of genome-wide association studies. PLoS Genet 2009; 5: e1000529.

45. Purcell, S. et al. PLINK: a toolset for whole genome association and population-based linkage analysis. Am J Hum Genet 81, 559-575 (2007).

46. R Development Core Team. Statistical Computing Software R: A language and environment for statistical computing, $R$ Foundation for Statistical Computing (2011).

47. SAS Institute Inc. SAS 9.4 Help and Documentation, Cary, NC, USA.

48. McLaren, L. Socioeconomic status and obesity. Epidemiol Rev 29, 29-48 (2007).

49. Beenackers, M. A. et al. Socioeconomic inequalities in occupational, leisure-time, and transport related physical activity among European adults: a systematic review. Int J Behav Nutr Phys Act 9, 116 (2012).

50. Junyent, M. et al. The effects of ABCG5/G8 polymorphisms on HDL cholesterol concentrations depend on ABCA1 genetic variants in the Boston Puerto Rican Health Study. J Lipid Res 50, 565-573 (2009).

51. Lu, Y. et al. Dietary n-3 and n-6 polyunsaturated fatty acid intake interacts with FADS1 genetic variation to affect total and HDLcholesterol concentrations in the Doetinchem Cohort Study. Am J Clin Nutr 92, 258-265 (2010).

52. Kim, D. S. et al. Novel gene-by-environment interactions: APOB and NPC1L1 variants affect the relationship between dietary and total plasma cholesterol. J Lipid Res 54, 1512-1520 (2013).

53. Gauderman, W. J. et al. Update on the state of the science for analytical methods for gene-environment interactions. Am J Epidemiol 186, 762-770 (2017)

54. Schultz, W. M. et al. Socioeconomic status and cardiovascular outcomes: challenges and interventions. Circulation 137, 2166-2178 (2018).

55. Marmot, M. et al. Closing the gap in a generation: health equity through action on the social determinants of health. Final report of the Commission on Social Determinants of Health. World Health Organization, Commission on Social Determinants of Health.

56. Marmot, M. et al. Fair society, healthy lives. The Marmot Review. Strategic Review of Health Inequalities in England post-2010, https://www.parliament.uk/globalassets/documents/fair-society-healthy-lives-full-report.pdf. Accessed 18 Mar 2021.

57. Zeng, L. et al. Genetically modulated educational attainment and coronary disease risk. Eur Heart J 40, 2413-2420 (2019). 
58. Cao, M. \& Cui, B. Association of educational attainment with adiposity, type 2 diabetes, and coronary artery diseases: a mendelian randomization study. Front Public Health 8, 112 (2020).

59. Krieger, N., Williams, D. R. \& Moss, N. E. Measuring social class in US public health research: concepts, methodologies, and guidelines. Annu Rev Public Health 18, 341-378 (1997).

60. Borghol, N. et al. Associations with early-life socio-economic position in adult DNA methylation. Int J Epidemiol 41, 62-74 (2012).

61. McGuinness, D. et al. Socio-economic status is associated with epigenetic differences in the pSoBid cohort. Int J Epidemiol 41, 151-160 (2012).

62. Fliesser M, De Witt Huberts J, Wippert P-M. The choice that matters: the relative influence of socioeconomic status indicators on chronic back pain- a longitudinal study. BMC Health Serv Res; 17. Epub ahead of print 2 December 2017. doi: https://doi.org/10. 1186/s12913-017-2735-9.

\section{Acknowledgements}

We are indebted to all study participants and to both the dedicated personnel of the study center of the Heinz Nixdorf Recall study and to the investigative group, in particular to U. Slomiany, E. M. Beck, A. Öffner, S. Münkel, R. Peter, and H. Hirche. Advisory Board: Meinertz T., Hamburg, Germany (Chair); Bode C., Freiburg, Germany; deFeyter P. J., Rotterdam, Netherlands; Güntert B, Halli, Austria; Gutzwiller F., Bern, Switzerland; Heinen H., Bonn, Germany; Hess O., Bern, Switzerland; Klein B., Essen, Germany; Löwel H., Neuherberg, Germany; Reiser M., Munich, Germany; Schmidt G., Essen, Germany; Schwaiger M., Munich, Germany; Steinmüller C., Bonn, Germany; Theorell T., Stockholm, Sweden; Willich S. N., Berlin, Germany.

\section{Author contributions}

$\mathrm{BS}$ formed the concept of the work. ND, RE and $\mathrm{KHJ}$ contributed to data acquisition. CE performed the statistical analysis and drafted the manuscript. MN performed sequencing of the genetic data. All authors contributed to the interpretation of results, revised the paper draft and approved the final manuscript.

\section{Funding}

Open Access funding enabled and organized by Projekt DEAL. This work was supported by the Heinz Nixdorf Foundation; the German Ministry of Education and Science [Grants Nationales Genomforschungsnetz, 01GS0820], the German Research Council [Projects SI 236/8-1, SI 236/9-1].

\section{Competing interests}

The authors declare no competing interests.

\section{Additional information}

Supplementary Information The online version contains supplementary material available at https://doi.org/ 10.1038/s41598-021-95970-Z.

Correspondence and requests for materials should be addressed to C.E.

Reprints and permissions information is available at www.nature.com/reprints.

Publisher's note Springer Nature remains neutral with regard to jurisdictional claims in published maps and institutional affiliations.

(c) (i) Open Access This article is licensed under a Creative Commons Attribution 4.0 International License, which permits use, sharing, adaptation, distribution and reproduction in any medium or format, as long as you give appropriate credit to the original author(s) and the source, provide a link to the Creative Commons licence, and indicate if changes were made. The images or other third party material in this article are included in the article's Creative Commons licence, unless indicated otherwise in a credit line to the material. If material is not included in the article's Creative Commons licence and your intended use is not permitted by statutory regulation or exceeds the permitted use, you will need to obtain permission directly from the copyright holder. To view a copy of this licence, visit http://creativecommons.org/licenses/by/4.0/.

(c) The Author(s) 2021 